\title{
A Cellular Automata Model for Heterogeneous Traffic Flow Incorporating Micro Autonomous Vehicles
}

\author{
Muhammad Tanveer $\mathbb{D}^{1},{ }^{1}$ Faizan Ahmad Kashmiri, ${ }^{2}$ Huimin Yan, ${ }^{1}$ Tianshi Wang, \\ and Huapu Lu ${ }^{1}{ }^{1}$ \\ ${ }^{1}$ Department of Civil Engineering, Tsinghua University, Beijing 100084, China \\ ${ }^{2}$ Department of Civil and Environmental Engineering, The Hong Kong University of Science and Technology, Hong Kong, China
}

Correspondence should be addressed to Huapu Lu; luhp@mail.tsinghua.edu.cn

Received 12 September 2020; Revised 8 November 2021; Accepted 10 December 2021; Published 28 January 2022

Academic Editor: Rakesh Mishra

Copyright ( 92022 Muhammad Tanveer et al. This is an open access article distributed under the Creative Commons Attribution License, which permits unrestricted use, distribution, and reproduction in any medium, provided the original work is properly cited.

\begin{abstract}
Despite the fact that significant research efforts have been made to the traffic flow theory of autonomous vehicles and manual vehicles, few existing studies have incorporated different modes of both vehicles in their analysis. In this study, we develop a cellular automata simulation model to investigate the impact of different modes of autonomous vehicles (autonomous car, autonomous bus, and autonomous micro car) and conventional vehicles (manual car, manual bus, and manual micro car) on the characteristics of traffic flow. A new type of autonomous mode, i.e., autonomous micro car, is investigated in the model to study the effects of this vehicle mode on the overall capacity of the network. Furthermore, two types of lane-changing behavior, i.e., aggressive lane changing and polite lane changing, are incorporated into the model. The results reveal that micro cars (manual and autonomous) have the potential to reduce traffic congestions and increase the capacity or flow rate (vehicles/hour) of the road. Where the average vehicle occupancy is less than 2 , if autonomous micro cars are deployed alongside autonomous cars, the flow rate (vehicles/hour) can be increased significantly. The results highlight the significance of the autonomous micro cars to traffic flow, passenger occupancy, and road capacity.
\end{abstract}

\section{Introduction}

Traffic congestion is a problem which is severely affecting daily commutes. The possible implications of conventional vehicles in terms of density, capacity, level of service, delay, peaks in fuel prices, environmental and pollution issues, parking issues, policies on taxation, etc. have been thoroughly investigated in the modern scenarios. Such complexities have forced the community to transition from conventional vehicular modes to alternative ones. There are several problems that are currently faced by conventional vehicles [1]. Although a conventional car can carry at most 5 people while one micro car caries 2 , the average occupancy is usually less than 2 . In the United States, car occupancy has fallen from 1.95 in 1960 to $1.38 \mathrm{p}-\mathrm{km} / \mathrm{v}-\mathrm{km}$. In Japan, it is even lower than in the US, having fallen from 1.45 in 1990 to $1.39 \mathrm{p}-\mathrm{km} / \mathrm{v}-\mathrm{km}$ in 2009. Hence, the carrying capacity can be ignored, especially in high motorized countries [2]. The term "micro car" describes a class of vehicle, generally two-door, two-seater, and less than 3 meters in length $[1,3]$.

With the recent advancements in computation and modern technology, the concept of autonomous cars is currently being tested out in multiple scenarios, and their explicit effects on traffic characteristics (for instance, flow rate, average speed, level of service, density, and capacity) are thoroughly investigated [4-6]. The standard autonomous car has the potential to reduce traffic congestions and increase the capacity of transportation networks due to their less reaction delay and coordinated patterns with one another [7-9]. If alongside the standard autonomous car a micro autonomous car is introduced, it may increase the capacity of any network further due to their smaller size and same reaction parameters. Additionally, the average 
vehicle occupancy of cars is less than 2 which means that the car is being underutilized. People prefer to drive their own vehicle than using other modes which subsequently increases the traffic congestion. If the average vehicle occupancy of any network is less than 2, then any additional arriving car with equal to or less than 2 passengers is further deteriorating the traffic flow rate. In the future, when autonomous drivers are running on road, then it will be difficult to resolve this issue through automation alone. It is assumed that shared mobility in autonomous vehicles will reduce traffic congestion considerably but ridesharing alone is not the only solution. If the personal ownership of autonomous cars increases, then the problem of congestion will rise again.

Thus, the goal of this research is to introduce a new type of autonomous vehicle, i.e., autonomous micro car in the transportation network, explicitly study its impact on traffic flow characteristics, and explore the characteristics of heterogeneous traffic flow of different types of conventional and autonomous vehicles, including capacity and fundamental diagrams. Since no autonomous micro cars are running on the road, a simulation model is developed to control the input parameters in order to highlight the importance of considering autonomous micro cars on roads. A simulation model thus gives us the ability to pinpoint the significance of an individual entity. Since our primary concern is the impact of individual vehicles on traffic flow characteristics, we chose the mesoscopic simulation model, i.e., cellular automata (CA), in this study to understand the effect of various vehicle types on overall traffic flow dynamics. More precisely, a new CA model is developed to simulate the traffic patterns of different types of autonomous and conventional vehicles in a one-way urban road segment. Additionally, different types of lane-change behavior for both autonomous and manual vehicles are introduced in this study to duplicate the realtime traffic patterns. Based on the simulation results, the importance of passenger occupancy is also highlighted by comparing the average vehicle occupancy of different countries.

The remainder of this paper is structured as follows. The second part of the paper is composed of literature review. The third part describes the model development. The results and discussion are meticulously explained in the fourth part, and the fifth part presents the concluding remarks.

\section{Literature Review}

Multiple traffic simulation techniques are used to model the traffic flow dynamics. We have developed a cellular automata (CA) model to capture the intricate details in traffic movements for this study. There are several characteristics relating to the perception and reaction of autonomous vehicles which differ from manual vehicles. As a result, the impact of autonomous vehicles on capacity is different from manual vehicles. The inclusion of micro cars in day-today flow dynamics can further change the capacity. Furthermore, the behavior of lane changing also plays a vital role in altering the flow rate. A brief review on all these topics is given below.

\section{Cellular Automata (CA) Model}

The CA model has been used extensively to research traffic stream behavior. It provides mathematical idealisations for physical systems and can be used for large-scale networks and has immense speed capability, even for traffic assignment and traffic forecasting purposes [10]. Complex traffic system seems to be simulated successfully by the CA model. Various models are developed to understand single-lane traffic, multilane traffic, lane-changing behavior, and network traffic situations [11]. Traffic phenomena such as the transition from free to congestion flow, lane inversion, and platoon formation can be accurately reproduced using CA model [2]. These models are able to evaluate vehicle traveling under real traffic situations due to the highly flexible transition rules and high computational efficiency [12].

Research on manual and autonomous vehicle mixed traffic flows is currently very minimal. Liu et al. carried out characteristics analysis of mixed traffic flow of AVs and manual vehicles using the CA model and found that the traffic capacity increases with the penetration of AVs and highlighted that overall traffic flow characteristics are mainly related to their smart maneuvers in lane changing and car following, and it seems that the car-following impact is more pronounced [5].

\section{Autonomous Vehicles Characteristics}

Autonomous vehicle drives without the human intervention. Autonomous driving differs from manual driving in a number of parameters such as acceleration, headway, speed during the lane-change maneuver, etc.

The primary effect of autonomous driving is the distribution of their perception-reaction time. Talebpour and Mahmassani found that the reaction time of intelligent connected vehicles (ICVs) is $50 \%$ less than that of regular vehicles [13]. Jin and Orosz defined the driver reaction time $(0.5-1 \mathrm{sec})$ or the sensing delay of the automated system $(0.05-0.2 \mathrm{sec})$ [14]. Wang et al. modified the reaction time of ICVs to $0.2 \mathrm{sec}$, while the reaction time of humans is $0.8 \mathrm{sec}$ [15]. Green studied the adaptive cruise control (ACC) response time, which is of the order of 0.1 to $0.2 \mathrm{sec}$ and is more negligible compared with the human reaction time of approximately $1 \mathrm{sec}$ [16]. Dixit et al. in their study showed that the reaction times to take control of the vehicle in the event of disengagement were found to have a stable distribution across different companies at $0.83 \mathrm{sec}$ on average [17]. However, there were differences observed in reaction times based on the type of disengagements, roadway, and autonomous miles travelled.

Studies have proven that automated driving can decrease traffic congestion by reducing the time headway, enhancing the traffic capacity, and improving the safety margins in car following [18]. Wang et al. in their study specified the minimum headway of ICV to be $0.8 \mathrm{~s}$ whereas the minimum headway of manual vehicles is $2 \mathrm{~s}$ [15]. Wietholt and Harding assumed the value of 0.4 with a standard deviation of 0 because the need for safety was considered in the study [19]. Gouy conducted a series of driving simulations to examine 
the effects of short headway. They accepted the headway of $0.3 \mathrm{sec}$ and $1.4 \mathrm{sec}$ for manual vehicle and ICV [20]. Vander Werf et.al. considered adopting ACC vehicles headway of $1.4 \mathrm{sec}$, and cooperative adaptive cruise control (CACC) vehicles headway of $0.5 \mathrm{sec}$ [21].

\section{Effect of Autonomous Vehicles on the Capacity}

Multiple studies have been done to showcase the effects of autonomous vehicles on highway capacity and reduction in traffic congestion. A group of autonomous cars can move at a greater density for any given speed than "normal cars," thereby increasing the capacity of roads $[7,8]$. Tientrakool et al. in their study showed that if all of the vehicles use both sensors and vehicle-to-vehicle communication, the increase in highway capacity is about $273 \%$ while if the vehicles use sensors alone, then increase in highway capacity is 43\% [9]. Van den Berg and Verhoef have given an overview of research on the effect of autonomous cars on capacity. All results refer to switching from $100 \%$ normal cars to $100 \%$ autonomous cars [8]. Liu et al. studied the mixed traffic flow with AVs and regular vehicles and found that the capacity increases with the penetration of AVs, and the effect of smart lanes shift in AVs on the general traffic flow characteristics is far less pronounced than smart car following [5]. Tanveer et al. studied the impact of including autonomous and manual buses alongside autonomous and manual cars. All the vehicles had distinct attributes. The results showed that autonomous buses can increase the capacity significantly if run alongside autonomous cars. Moreover, more passengers can be accommodated by inclusion of autonomous buses [22]. Similarly, Muhammad et al. studied the impact of autonomous vehicles in a heterogeneous environment where manual vehicles were categorized on the basis of age and gender. The results showed that autonomous vehicles could significantly increase traffic flow even among drivers with different attributes [23].

\section{Micro Cars}

Due to rising traffic safety and congestion issues, the concept of micro cars is being recognized again. As stated earlier, a micro car is less than three meters in length, lightweight, usually a two-seater, and with two doors $[1,3]$. The introduction of the mini/micros will certainly increase steadystate flow capacity and reduce headways, making it likely that tailgating accidents will increase [24]. Mu and Yamamoto studied the characteristics of traffic with micro cars in traffic flow by using a cellular automata simulation model on both a highway segment and arterial segment with two lanes, including an intersection delay with a signal cycle in the middle of the latter. The results demonstrate that the introduction of micro cars will relieve traffic congestion to some extent, and vehicles passing through per hour become more than those without micro cars [2]. Mu and Yamamoto [25] also calculated the energy consumption of traffic on both a highway segment and an arterial segment with a single traffic light at its midpoint for different ratios of micro cars. From the results, the environmental effects of micro cars were analysed. Those two studies are a prediction of micro cars effect on assumed road segments, not traffic network. $\mathrm{Mu}$ and Yamamoto simulated the traffic simulations through VISSIM and found out that introduction of micro cars reduces total network travel time if their desired speed is set at $48 \mathrm{~km} / \mathrm{h}$ to $58 \mathrm{~km} / \mathrm{h}$ or $40 \mathrm{~km} / \mathrm{h}$ to $45 \mathrm{~km} / \mathrm{h}$. The reduction is greater if the two vehicle types have the same desired speed [3].

\section{Lane-Change Behavior}

Gipps proposed the structure for lane-changing model based upon a deterministic rule [26]. Yang and Koutsopoulos modified the model by adding a certain probability in each lane-change maneuver to mimic driver's uncertainty [27]. Hidas proposed the simulation of intelligent transport system to evaluate the intelligent transportation systems application such as public transport preference, congestion, and dynamic route guidance and the results indicate that, during congested conditions, only forced models with cooperative lane change can generate realistic flow-speed relationships [28].

Hidas developed the structure of a lane-changing model for urban and highways to capture lane-changing vehicle interaction, which was then in three classes, i.e., free, cooperative, and forced lane change based on the video recording observation. He analysed the intelligent agent-based simulation model techniques to improve overall performance and reliability in the dynamic traffic scenario [28]. Kesting et al. developed the minimum braking model, i.e., MOBIL (Minimizing Overall Braking Induced by Lane changes) model for modeling lane-change decisions [29]. Car following (CF) and lane changing (LC) are two primary driving tasks observed in traffic flow and are thus vital components of traffic flow theories [30]. Generally, lanechange behavior could be classified as either discretionary or mandatory according to driving incentives [31]. LC did not receive much attention until recently. This is because of the increasing evidence of (1) LC's negative impact on traffic safety and (2) its linkage to macroscopic traffic flow characteristics [30]. Zheng has comprehensively reviewed recent developments in modeling lane-changing behavior. The study concluded that there is a clear need to develop a comprehensive model that captures the (mandatory or discretionary) LC decision-making process and its significant impact on neighboring traffic [30]. Multiple studies have been conducted to analyse the lane-change behavior on freeway weaving segments through numerous aspects, mainly including empirical analyses of lane-change characteristics and lane-change decision modeling [32]. Liu et al. analysed the impact of AVs on the traffic flow characteristic and lane-change frequency [5].

This paper developed the CA model for the heterogeneous traffic flow. The previous studies on autonomous vehicles did not cover the concept of heterogeneous traffic flow dynamics in terms of different types of autonomous and manual vehicles. Diverse types of autonomous vehicles have the potential to have distinct effects on traffic patterns. As 
mentioned before, this novel study is one of the pioneers, simulating traffic with autonomous micro cars through CA modeling, which can simulate nonlinear and complex traffic flow dynamics. Furthermore, it is the first trial to simulate traffic flow with autonomous micro cars in a small one-way urban traffic network. Moreover, different lane-changing methodologies have been adopted for autonomous and manual vehicles as the concept of automation with efficient lane-changing patterns in the vehicular technology has excellent future opportunities. Thus, it is essential to understand that impact of different modes of autonomous and manual vehicles on overall traffic dynamics. A detailed description of the CA model developed for simulating heterogeneous traffic is presented. The heterogeneous traffic involves vehicles with numerous static and dynamic features.

\section{Model Development}

Many people have acknowledged having a car in their lives and it has become a staple of society today. While commuting in public transport, they are likely to use their own car. A micro car is easy to drive and park, has lane-change manoeuvre and better gas mileage, is economically cheaper, and significantly influences road capacity compared to a regular car. Since micro cars are different from the traditional cars, there are significant differences between traffic flows with and without micro cars in terms of maximum speed, acceleration, and dimension. This paper describes the CA principle in modeling mixed traffic flows, including micro vehicles. This model simulates heterogeneous flows of MVs and AVs using an advanced CA model. The objectives of our CA model are (i) to analyse the impact of the autonomous vehicle under the mixed traffic condition, (ii) analyse impact of car size on the road capacity, (iii) investigate the car following behavior, and (iv) investigate the lane-change behavior of different types of vehicle. In order to capture the real traffic scenario, we have developed a new 3 lane freeway CA model for one-way urban network. In this model, we have introduced six different types of vehicles, i.e., micro autonomous car (MA), micro manual car (MM), autonomous car (AC), manual car (MC), autonomous bus $(\mathrm{AB})$, and manual bus (MB). These vehicles have different attributes such as speed, headway, size, reaction time, and lane-change behavior. We have tried to explore the impact of mixed autonomous vehicles on traffic flow characteristics and explicitly the influence of micro vehicles on traffic flow rate with pivotal focus on micro autonomous car. By understanding the significance of MA in a network, the importance of its inclusion in the near future alongside $\mathrm{AC}$ and $\mathrm{AB}$ should be addressed. In order to simulate the complex traffic dynamics of each vehicle, CA model provides flexibility in real-time traffic situations with different vehicle features that are the foundation of this research, as both manual and autonomous vehicles will have different traits. All the previous traffic flow models have not introduced the concept of heterogeneous autonomous vehicles. This study does that and introduces new types of autonomous mode, i.e., $\mathrm{MA}, \mathrm{AC}$, and $\mathrm{AB}$, in a transportation system that will be highly beneficial in assuaging traffic congestion problems.

Table 1 addresses all symbols and abbreviations presented in this study.

In order to understand how the model works, it is essential to understand the following terminologies.

The minimum headway is defined as

$$
\begin{aligned}
D_{\mathrm{nmm}} & =x_{i+1}-x_{i}-1, \\
D_{\mathrm{nma}} & =x_{i+1}-x_{i}-1, \\
D_{\mathrm{nmc}} & =x_{i+1}-x_{i}-2, \\
D_{\mathrm{nmc}} & =x_{i+1}-x_{i}-2, \\
D_{\mathrm{nmb}} & =x_{i+1}-x_{i}-4, \\
D_{\mathrm{nmb}} & =x_{i+1}-x_{i}-4 .
\end{aligned}
$$

The deterministic acceleration of every vehicle is defined as

$$
\begin{aligned}
& \text { acceleration } v_{n, \mathrm{~mm}} \longrightarrow \min \left(v_{\mathrm{max}, \mathrm{mm}}, v_{n, \mathrm{~mm}}+1\right), \\
& \text { acceleration } v_{n, \mathrm{ma}} \longrightarrow \min \left(v_{\mathrm{max}, \mathrm{ma}}, v_{n, \mathrm{ma}}+1\right), \\
& \text { acceleration } v_{n, \mathrm{mc}} \longrightarrow \min \left(v_{\mathrm{max}, \mathrm{mc}}, v_{n, \mathrm{mc}}+1\right), \\
& \text { acceleration } v_{n, \mathrm{ac}} \longrightarrow \min \left(v_{\max , \mathrm{ac}}, v_{n, \mathrm{ac}}+1\right), \\
& \text { acceleration } v_{n, \mathrm{mb}} \longrightarrow \min \left(v_{\max , \mathrm{mb}}, v_{n, \mathrm{mb}}+1\right), \\
& \text { acceleration } v_{n, \mathrm{ab}} \longrightarrow \min \left(v_{\max , \mathrm{ab}}, v_{n, \mathrm{ab}}+1\right) .
\end{aligned}
$$

All the vehicles accelerate according to the aforementioned rules as their position is updated at each time step. Similarly, the deterministic deceleration of every vehicle is defined as

$$
\begin{aligned}
& \text { deterministic deceleration } v_{n, \mathrm{~mm}} \longrightarrow \min \left(v_{n, \mathrm{~mm}}, D_{\mathrm{nmm}}\right), \\
& \text { deterministic deceleration } v_{n, \mathrm{ma}} \longrightarrow \min \left(v_{n, \mathrm{ma}}, D_{\mathrm{nma}}\right), \\
& \text { deterministic deceleration } v_{n, \mathrm{mc}} \longrightarrow \min \left(v_{n, \mathrm{mc}}, D_{\mathrm{nmc}}\right), \\
& \text { deterministic deceleration } v_{n, \mathrm{ac}} \longrightarrow \min \left(v_{n, \mathrm{ac}}, D_{\mathrm{nac}}\right), \\
& \text { deterministic deceleration } v_{n, \mathrm{mb}} \longrightarrow \min \left(v_{n, \mathrm{mb}}, D_{\mathrm{nmb}}\right), \\
& \text { deterministic deceleration } v_{n, \mathrm{ab}} \longrightarrow \min \left(v_{n, \mathrm{ab}}, D_{\mathrm{nab}}\right) .
\end{aligned}
$$

The position update of each vehicle is defined as

$$
\begin{gathered}
x_{n, \mathrm{~mm}}=x_{n, \mathrm{~mm}}+v_{n, \mathrm{~mm}}, \\
x_{n, \mathrm{ma}}=x_{n, \mathrm{ma}}+v_{n, \mathrm{ma}}, \\
x_{n, \mathrm{mc}}=x_{n, \mathrm{mc}}+v_{n, \mathrm{mc}}, \\
x_{n, \mathrm{ac}}=x_{n, \mathrm{ac}}+v_{n, \mathrm{ac}}, \\
x_{n, \mathrm{mb}}=x_{n, \mathrm{mb}}+v_{n, \mathrm{mb}}, \\
x_{n, \mathrm{ab}}=x_{n, \mathrm{ab}}+v_{n, \mathrm{ab}} .
\end{gathered}
$$


TABLE 1: Symbols and abbreviations.

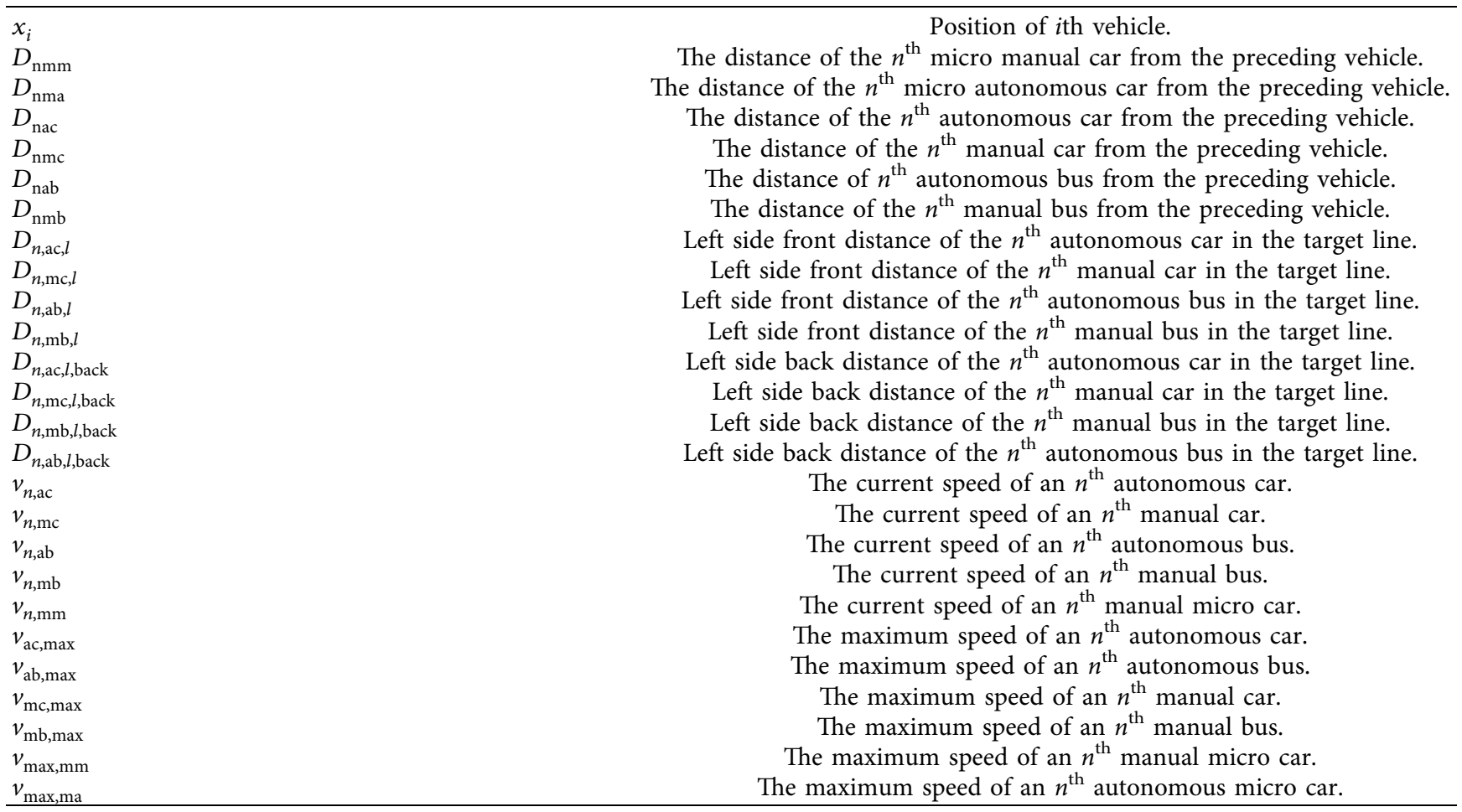

8.1. Lane-Changing Rules. We also have developed different lane-changing policies for both manual and autonomous vehicles to simulate real-time traffic maneuvers with different vehicles. We also introduced a probability factor for lane-change maneuver, as in some cases vehicles may not choose to switch lanes even if the condition is in their favor. Furthermore, for the lane change, the front distance of vehicles in the target lane should be more than the minimum safe distance (headway). There are two different types of lane-change behavior of drivers, i.e., polite lane change (PLC) and aggressive lane change (ALC). All types of autonomous vehicles will follow both the aggressive and polite lane-change rules in the model. In contrast, all types of manual vehicles will follow the aggressive lane-change rules $[33,34]$. In general, manual drivers are not patient and calculating as the computerized vehicles. They will adjust their lanes in such a manner that they get the most benefit as a way to reduce their travel time by traveling fast because for each manual driver their travel time and ease in driving are most important to them so they can change lanes without considering the impact on system wide network. The autonomous vehicles on the other hand can switch their mode according to situation. They can appraise the impact of their lane-changing behavior on other vehicles and be able to mimic the aggressiveness of human drivers if conditions require. However, this may not be applicable in real-time scenarios where the priority of each AV is safety and reliability. Lane change is acceptable in any direction. In the model, the first preference is given to left lane change. However, if the condition does not match, then right lanechange rules is acceptable. If the vehicles cannot change lanes in either direction, they adjust their speed and keep in the same lane. There is another restriction on the buses such that both $\mathrm{AB}$ and $\mathrm{MB}$ will move in the first and second lane only, and cars can occupy any lane to simulate real-world traffic maneuvers. Both the right lane change and left change rules have similar nomenclature.

8.2. Polite Lane-Change Rules. For any vehicle which is going to consider the lane change from the left side, the following criteria are checked first. (a) For any $n$ vehicles, the vehicle will first reach the minimum safe distance by increasing the speed. (b) After reaching the minimum safe distance, the front distance in the target lane is checked. (c) Finally, in order to change lane, the speed of that $n$ vehicle should be higher than the back vehicle in the target lane.

$$
\begin{aligned}
D_{n, \text { an }, v} & <\min \left(v_{n, \text { an }, v}(t)+1, v_{\max , \mathrm{an}, v}\right), \\
D_{n, l} & >D_{n, \mathrm{an}, v}, \\
D_{n, l, \mathrm{back}} & >v_{\text {max,an, } v} .
\end{aligned}
$$

Here, $v_{\text {max,an, } v}$ represents the maximum speed of any vehicle and $D_{n \text {,an, } v}$ represents the front distance of any vehicle in the traveling lane. Similar nomenclature of polite lane change applies for the right-lane-change case. If the vehicle does not meet the criteria of left change rules, then the vehicle will check the possibility of right lane change.

$$
\begin{gathered}
D_{n, r}>D_{n, \text { an }, v}, \\
D_{n, r, \text { back }}>v_{\text {max,an }, v} .
\end{gathered}
$$


8.3. Aggressive Lane-Change Rules. Unlike polite lanechange rules, for any $n$ vehicles, the vehicle does not need to reach the minimum safe distance by increasing the speed. We have introduced probability factor for aggressive lanechange maneuver, as in some cases vehicles may not choose to switch lanes even if the condition is in their favor. It can change lane even if the distance from the preceding vehicle in the traveling lane is greater than the minimum safe distance. For instance, in the case of MM, if the front and back distance in the target lane are 4 cells and speed is higher than the back vehicle in the target lane, then MM can follow the aggressive lane behavior. This is similar for MA, AC, MC, $\mathrm{MB}$, and $\mathrm{AB}$.

Figure 1 shows the schematic diagram of the model with six different types of vehicles running on road.

8.4. Numerical Simulation. This proposed CA model is created by using MATLAB. This model focuses on the mixed traffic flow of the MVs and AVs. This model improves the existing CA model to capture the difference between MVs and AVs. The simulation is employed to analyse the traffic flow characteristics variation in mixed traffic flow under different penetration rates of AVs (MA, $A C$, and $A B$ ) with manual vehicles ( $M M, M C$, and $M B)$, reaction time, and lane-change behavior. As mentioned before, each vehicle is defined by its own class. The class has several attributes: ID, length, traveling lane, back-space distance, front-space distance, position, speed, maximum speed, delay, and type. As shown in Figure 1, a freeway stretch of 3 lanes without on/off-ramps was considered for simulation studies. $L$ represents each lane, and total length of freeway is 10,000 meters. The cell size is 3 meters. The size of MA and MM is equal to 1 cell; the size of AC and MC is 2 cells, whereas the size of $\mathrm{AB}$ and $\mathrm{MB}$ is kept as 4 cells. Further explanation is provided in Pseudocode 1 . The speed of any MA, MM, AC, and MC vehicle is at one of the eight discrete levels, which are 0-7 cells per time step. "0" means that the vehicle is not moving, while " 7 " means the vehicle can travel across 7 cells within one-time step and the corresponding maximum speed is $80 \mathrm{~km} / \mathrm{h}$. In contrast, the speed of any $A B$ and $M B$ vehicle is maximum $60 \mathrm{~km} / \mathrm{h}$, which means it can travel 5 cells in one second. All the autonomous vehicles differ from their counterparts' manual vehicles in terms of headway, reaction delay, and lane-changing maneuvers. We have also implanted the random brake probability in all manual vehicles because the driver cannot maintain a constant speed.

\footnotetext{
\# Define the road parameter

Road length $=L$;

Max speed of line $=\mathrm{LV}_{\text {max }}$;

No. of lines $=L \mathrm{i}$

\# Define the vehicle parameter

Veh_ID;

Veh_position;

Veh_length;
}

Veh_Line;

Veh_speed;

Veh_max speed;

front space;

back space;

Veh_Delay;

Veh_headway.

\section{\# Define the randomization break}

Randomization $v_{n} \max \left(v_{n}-1,0\right)$

\# Define the position update

$X_{n}=X_{n}+v_{n}$

\# Define the deterministic acceleration

$v_{n} \longrightarrow \min \left(v_{\max }, v_{n}+1\right)$

\# Define the deterministic deceleration

$v_{n} \longrightarrow \min \left(v_{n}, D_{n}\right.$

\#Define the PLC_Prob, \& ALC_Prob

While (no. of veh $<=$ total no. of veh)

Time $=0$

Veh_speed $=0$

AvgSpeed $=0$

If (time<10000)

front space of veh $>$ veh. headway

Update position

Else

\# Polite Lane Change

P_Prob $=$ rand (1)

If (P_Prob $<$ PLC_Prob)

If (front space of veh $<=$ headway)

\# Check the either lane option

Speed of veh> speed of back veh in target lane

Space of veh $>$ front space in target lane

Update the position

Else

Decelerate --

\#Aggressive lane change probability

A_Prob $=$ rand (1)

If (A_Prob < ALC_Prob)

Speed of veh $>$ speed of back vehicle in target lane

Space of veh $>$ front space in target lane

Update the position

Else

Decelerate --

If (Veh_speed $\leq$ Veh_max speed \& Veh_speed $\leq$ Max speed of line)

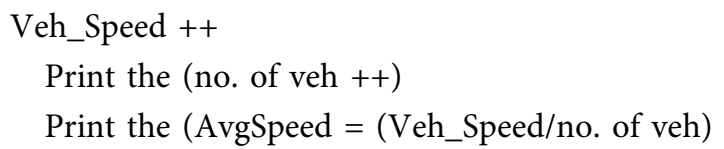




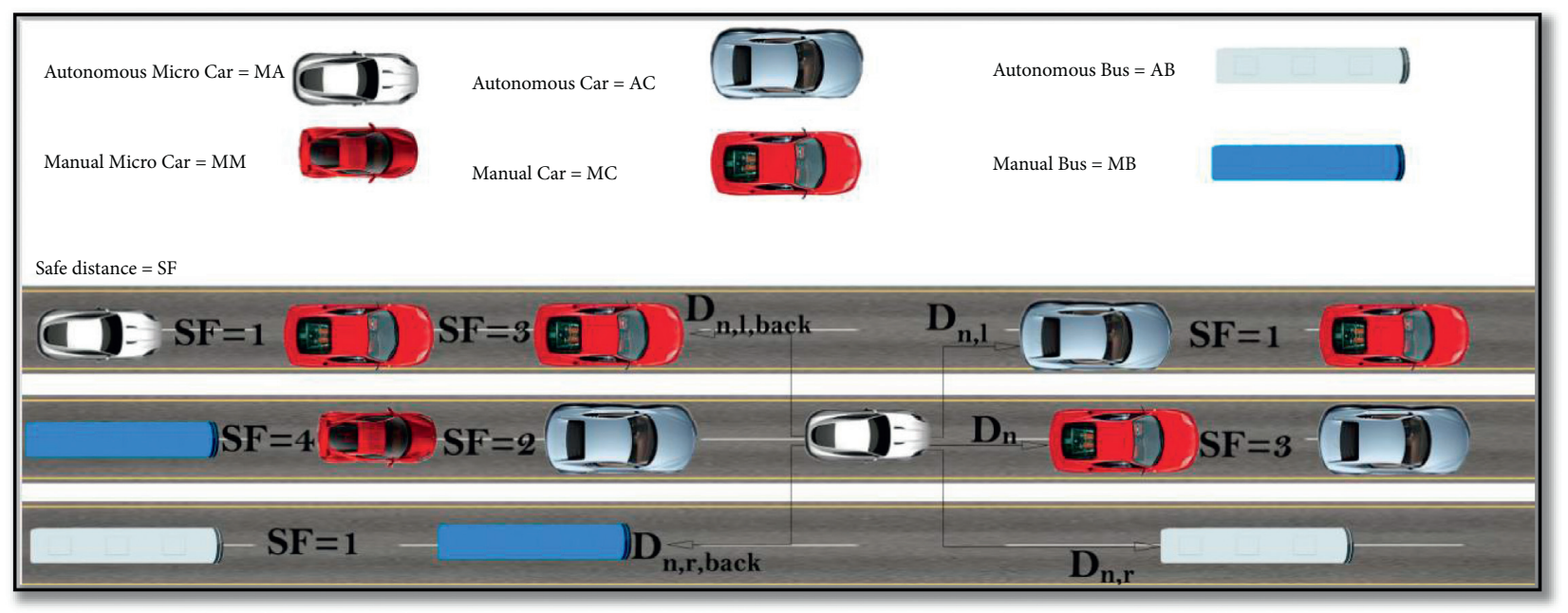

Figure 1: Schematic diagram of the model.

The minimum safe distance is defined as 1 cell for each autonomous vehicle due to its less reaction delay [4], where, for $\mathrm{MM}$, the minimum safe distance is 2 cell. For MC, the minimum safe distance is 3 cells and for $\mathrm{MB}$ the minimum safe distance is 4 cells. In order to shift to target lane, the minimum distance for the front (preceding vehicle) and back (following vehicle) for MA, AC, and AB is 2 cells. For MM, the minimum distance for lane change in the target lane is 3 cells distance for the front and back. For the MC, the minimum distance for lane change in the target lane is 4-cell distance for front and back. For MB, the minimum front and back distance for lane change are 5 cells. The vehicles are restricted to remain in the same lane if the distance in the target lane is not more than safe distance. Table 2 represents the front safe distance and lane-change distance for each vehicle.

Figure 2 describes the flow chart regarding lane-change behavior and decision in vehicles. Different combinations of the vehicles at different penetration rates and at different values of random brake, PLC, and ALC were simulated. All the scenarios include different penetration rates of $\mathrm{MM}$ and MA in order to highlight their significance in the network explicitly. The simulation was run for 1000 time steps for each scenario. The density $(k)$, speed $(\bar{v})$, and flow rate $(q)$ are determined by the following formula:

$$
\begin{aligned}
\bar{v} & =\frac{1}{T} \underset{\substack{T+t_{0}-1 \\
\mathrm{a}}}{\substack{\mathrm{a} \\
t=t_{0}}} \bar{N} \underset{n=1}{\mathrm{a}} v_{n}(t), \\
k & =\frac{N}{3 L}, \\
q & =k \cdot \bar{v} .
\end{aligned}
$$

\section{Results and Discussion}

As stated before, multiple setups with different composition of vehicles have been simulated in this study. The fundamental traffic characteristics, i.e., speed, density, and flow rate, have been computed of each composition in all scenarios. Based upon the simulation results, it is easy to highlight which combination performs efficiently in congested phase and free-flow phase. In scenarios 1 to 5 , the values of ALC and PLC are changed by keeping the traffic composition in each scenario constant to focus on the effect of lane-changing behavior of different vehicles on overall flow. The values of ALC and PLC range from 0.0 to 1.0 with 0.2 intervals. It is important to understand here the importance of lane-changing behavior under heterogeneous traffic conditions whether they significantly influence the overall capacity of a network or not. In scenarios 6 to 11, the different penetration rate of micro vehicles is introduced in each composition with a range from $20 \%$ to $80 \%$ with $20 \%$ step as shown in Table 3. In these scenarios, values of ALC and PLC are kept constant at 0.5 to underscore the impact of micro cars on overall traffic flow rate. The details of the scenarios are mentioned in Table 3.

9.1. Scenario 1. In this setup, the composition is the following:

$20 \%$ MA, 20\% MM, 20\% AC, 20\% MC, 10\% AB, $10 \%$ $\mathrm{MB}$ with different values of PLC (0.0 to 1.0) when ALC is kept constant at 0

Figures 3-5 present the traffic flow fundamental diagrams of scenario 1 . There are 5 different types of simulations in this scenario. From the figures, it is quite evident that as the aggressive behavior probability increases, the flow rate and average speed of the network increase under the same density. Since both manual and autonomous vehicles can change lanes aggressively, the capacity of the network increases due to the high percentage of lane-changing vehicles. When both ALC and PLC are kept at 0 , none of the vehicles in this simulation can change their lanes as there is no probability of changing the lane. The maximum flow rate in this setup is 2085 vehicles per hour and average maximum speed is $58.32 \mathrm{~km} / \mathrm{hr}$. In comparison, when ALC is 1 and PLC is 0, the maximum flow rate becomes 2457 vehicles per hour (around an $17.8 \%$ increase) and the average maximum speed is $62.89 \mathrm{~km} / \mathrm{hr}$ (around an $7.8 \%$ increase). Thus, 
TABLE 2: The front safe distance and lane-change distance for each vehicle.

\begin{tabular}{lcc}
\hline Vehicle & Safe distance & Front and back distance in the target lane \\
\hline MA & 1 cell & 2 cells \\
MM & 2 cells & 3 cells \\
AC & 1 cell & 2 cells \\
MC & 3 cells & 4 cells \\
MB & 4 cells & 5 cells \\
AB & 1 cell & 2 cells \\
\hline
\end{tabular}

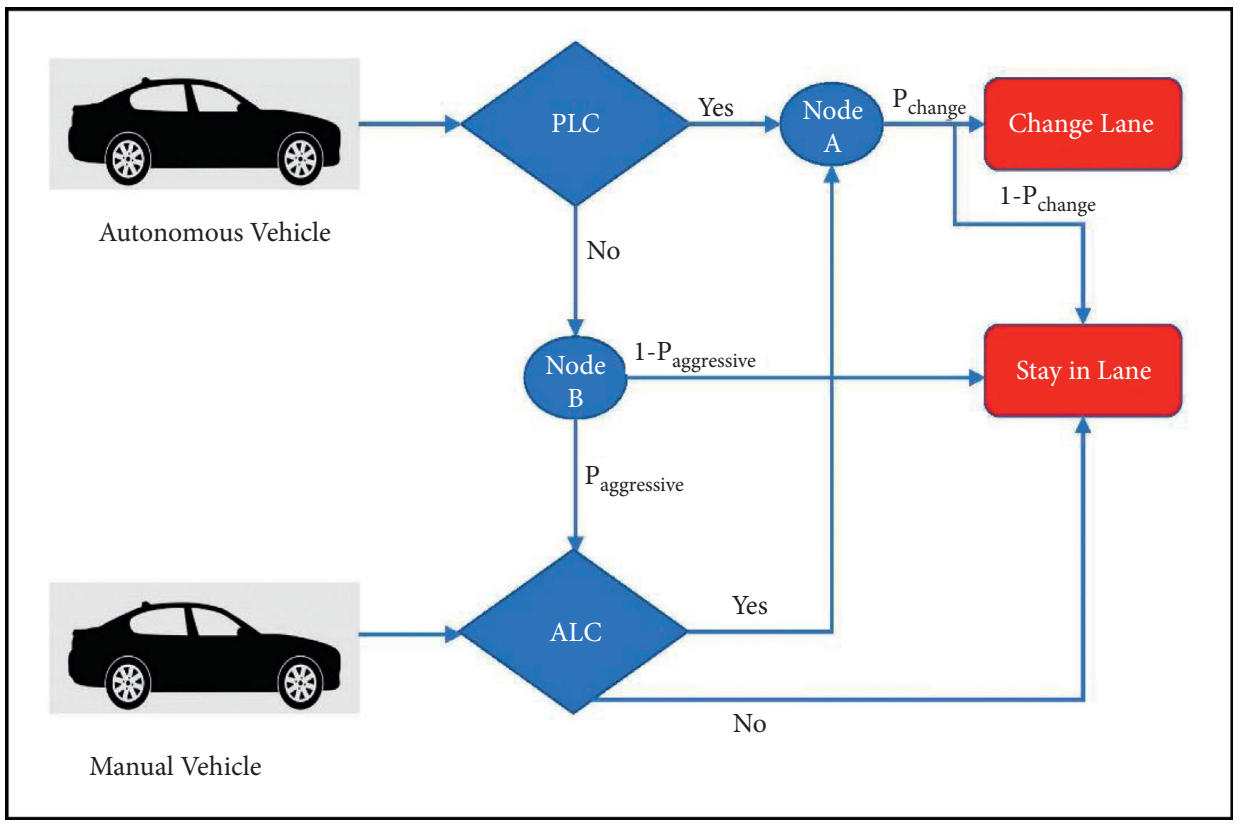

Figure 2: Flow chart of lane change.

TABle 3: Details of different scenarios.

\begin{tabular}{|c|c|}
\hline Scenarios & Details/composition in model \\
\hline Scenario 1 & $\begin{array}{l}20 \% \mathrm{MA}, 20 \% \mathrm{MM}, 20 \% \mathrm{AC}, 20 \% \mathrm{MC}, 10 \% \mathrm{AB}, 10 \% \mathrm{MB} \text { with different values of ALC (0.0 to 1.0) when PLC is kept constant } \\
\text { at } 0\end{array}$ \\
\hline Scenario 2 & $\begin{array}{l}20 \% \mathrm{MA}, 20 \% \mathrm{MM}, 20 \% \mathrm{AC}, 20 \% \mathrm{MC}, 10 \% \mathrm{AB}, 10 \% \mathrm{MB} \text { with different values of PLC (0.0 to } 1.0) \text { when ALC is kept constant } \\
\text { at } 0\end{array}$ \\
\hline Scenario 3 & $20 \% \mathrm{MA}, 20 \% \mathrm{MM}, 20 \% \mathrm{AC}, 20 \% \mathrm{MC}, 10 \% \mathrm{AB}, 10 \% \mathrm{MB}$ with different values of ALC $(0.2$ to 0.8$)$ and PLC (0.2 to 0.8$)$ \\
\hline Scenario 4 & $8 \% \mathrm{MA}, 36 \% \mathrm{MM}, 8 \% \mathrm{AC}, 36 \% \mathrm{MC}, 4 \% \mathrm{AB}, 8 \% \mathrm{MB}$ with different values of ALC $(0.2$ to 0.8$)$ and PLC (0.2 to 0.8$)$ \\
\hline Scenario 5 & $100 \% \mathrm{MA}$ and $100 \% \mathrm{MM}(\mathrm{PLC}$ and $\mathrm{ALC}=0.5)$ \\
\hline Scenario 6 & $20 \% \mathrm{MA}+80 \% \mathrm{MC}, 40 \% \mathrm{MA}+60 \% \mathrm{MC}, 60 \% \mathrm{MA}+40 \% \mathrm{MC}, 80 \% \mathrm{MA}+20 \% \mathrm{MC}(\mathrm{PLC}$ and $\mathrm{ALC}=0.5)$ \\
\hline Scenario 7 & $20 \% \mathrm{MA}+80 \% \mathrm{AC}, 40 \% \mathrm{MA}+60 \% \mathrm{AC}, 60 \% \mathrm{MA}+40 \% \mathrm{AC}, 80 \% \mathrm{MA}+20 \% \mathrm{AC}(\mathrm{PLC}$ and $\mathrm{ALC}=0.5)$ \\
\hline Scenario 8 & $20 \% \mathrm{MA}+80 \% \mathrm{MM}, 40 \% \mathrm{MA}+60 \% \mathrm{MM}, 60 \% \mathrm{MA}+40 \% \mathrm{MM}, 80 \% \mathrm{MA}+20 \% \mathrm{MM}(\mathrm{PLC}$ and $\mathrm{ALC}=0.5)$ \\
\hline Scenario 9 & $20 \% \mathrm{MM}+80 \% \mathrm{AC}, 40 \% \mathrm{MM}+60 \% \mathrm{AC}, 60 \% \mathrm{MM}+40 \% \mathrm{AC}, 80 \% \mathrm{MM}+20 \% \mathrm{AC}(\mathrm{PLC}$ and $\mathrm{ALC}=0.5)$ \\
\hline Scenario 10 & $20 \% \mathrm{MM}+80 \% \mathrm{MC}, 40 \% \mathrm{MM}+60 \% \mathrm{MC}, 60 \% \mathrm{MM}+40 \% \mathrm{MC}, 80 \% \mathrm{MM}+20 \% \mathrm{MC}(\mathrm{PLC}$ and $\mathrm{ALC}=0.5)$ \\
\hline Scenario 11 & $90 \% \mathrm{MM}+10 \% \mathrm{AB}, 90 \% \mathrm{MM}+10 \% \mathrm{MB}, 90 \% \mathrm{MA}+10 \% \mathrm{AB}, 90 \% \mathrm{MA}+10 \% \mathrm{AB}(\mathrm{PLC}$ and $\mathrm{ALC}=0.5)$ \\
\hline
\end{tabular}

aggressive lane-change behavior followed by any kind of vehicle can increase the flow rate significantly.

9.2. Scenario 2. In this setup, the composition is the following:

$20 \%$ MA, 20\% MM, 20\% AC, 20\% MC, 10\% AB, 10\% $\mathrm{MB}$ with different values of ALC (0.0 to 1.0) when PLC is kept constant at 0
Figures 6-8 present the traffic flow fundamental diagrams of scenario 2 . There are 5 different types of simulations in this scenario. From the figures, it is apparent that the increase in polite lane-changing behavior does not significantly impact the flow rate and average speed of the network under the same density. Even when the simulation in which PLC and ALC both are 0 is compared with the setup that the value of PLC is equal to 1 and ALC is equal to 0 , the increase in capacity of the road network is trivial. This is because, in the model, only autonomous vehicles are allowed to change 


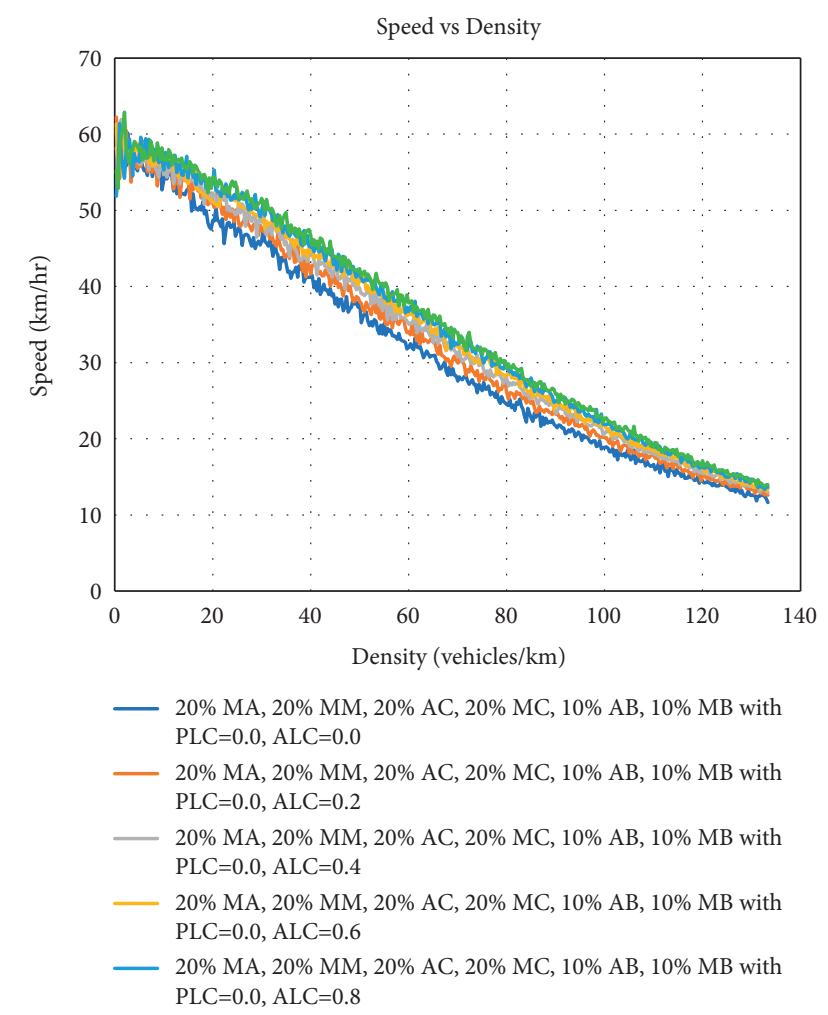

FIgURE 3: Speed vs. density fundamental diagrams for different compositions of $\mathrm{MA}, \mathrm{MM}, \mathrm{AC}, \mathrm{MC}, \mathrm{AB}$, and $\mathrm{MB}$ with different values of ALC when PLC is fixed at 0 .

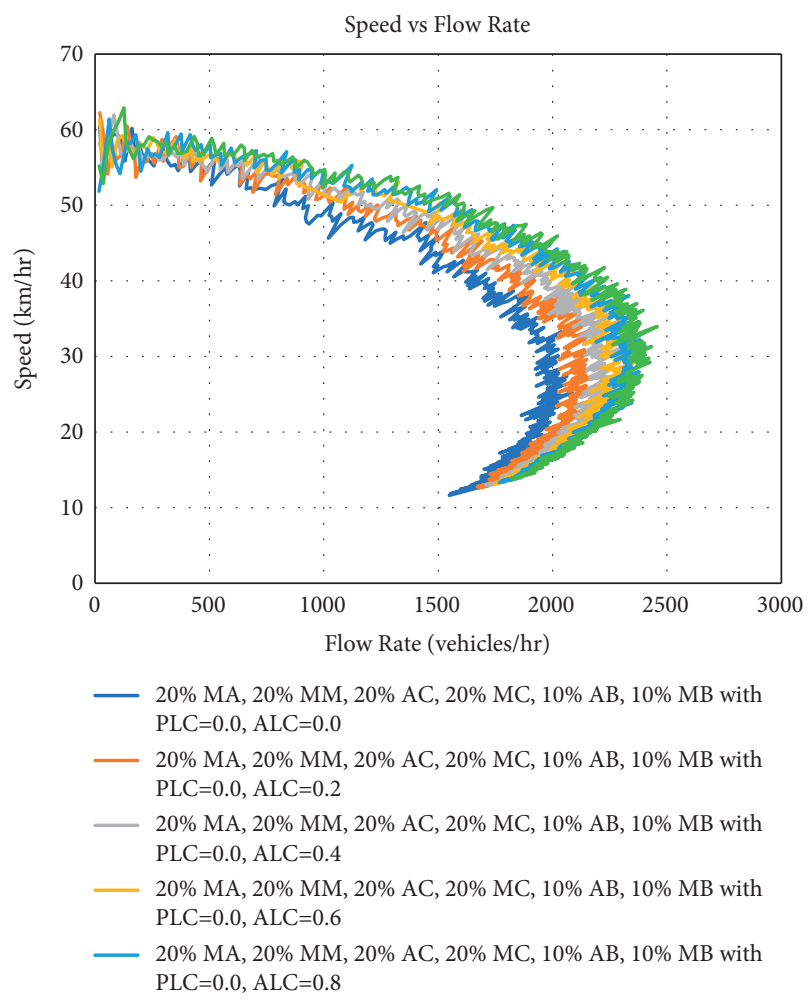

Figure 4: Speed vs. flow rate fundamental diagrams for different compositions of MA, MM, AC, MC, $\mathrm{AB}$, and $\mathrm{MB}$ with different values of ALC when PLC is fixed at 0 .

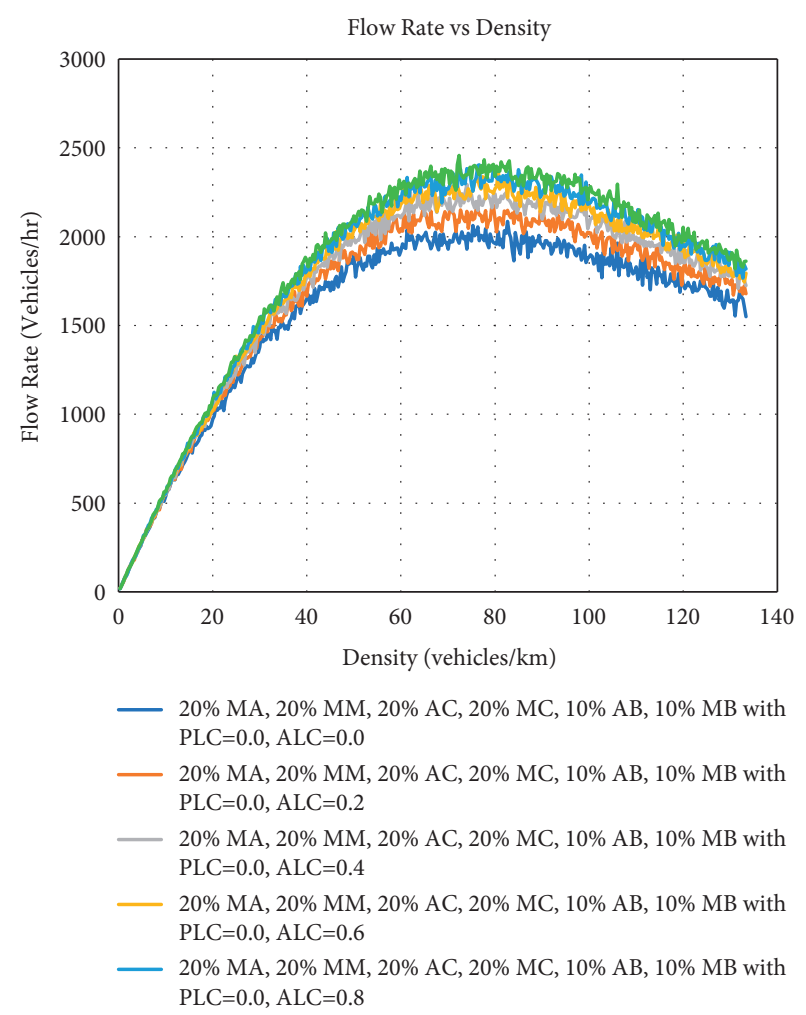

Figure 5: Flow rate vs. density fundamental diagrams for different compositions of MA, MM, AC, MC, $\mathrm{AB}$, and $\mathrm{MB}$ with different values of ALC when PLC is fixed at 0 .

lanes both aggressively and politely and manual vehicles will only change lanes under aggressive behavior. Since 50 percent of the vehicles in this scenario are manual, only the remaining $50 \%$ percent will be allowed to change lanes. Furthermore, when following polite lane-change behavior, autonomous vehicles when changing their lanes should not interfere with the movement of the neighboring vehicles in adjacent lanes unlike in aggressive lane-changing policy. Thus, polite lane-change behavior followed by an autonomous vehicle does not affect the flow rate significantly.

9.3. Scenario 3. In this setup, the composition is the following:

$20 \%$ MA, 20\% MM, 20\% AC, 20\% MC, $10 \%$ AB, $10 \%$ $\mathrm{MB}$ with different values of ALC (0.2 to 0.8$)$ and PLC (0.2 to 0.8$)$

Figures 9-11 present the traffic flow fundamental diagrams of scenario 3 . There are 4 different types of simulations in this scenario with different values of PLC and ALC in each one. It is clear from the figure that as soon as ALC values increase, the capacity and average speed of the network increase under the same density which aligns with results of our scenario 1 . When PLC is 0.8 and ALC is 0.2 , the flow rate values are less than others. The maximum flow rate value in this composition is 2207 vehicles per hour. In comparison, when PLC is 0.2 and ALC is 0.8 , the maximum flow rate is 2417 vehicles per hour $(9.5 \%$ increase in 


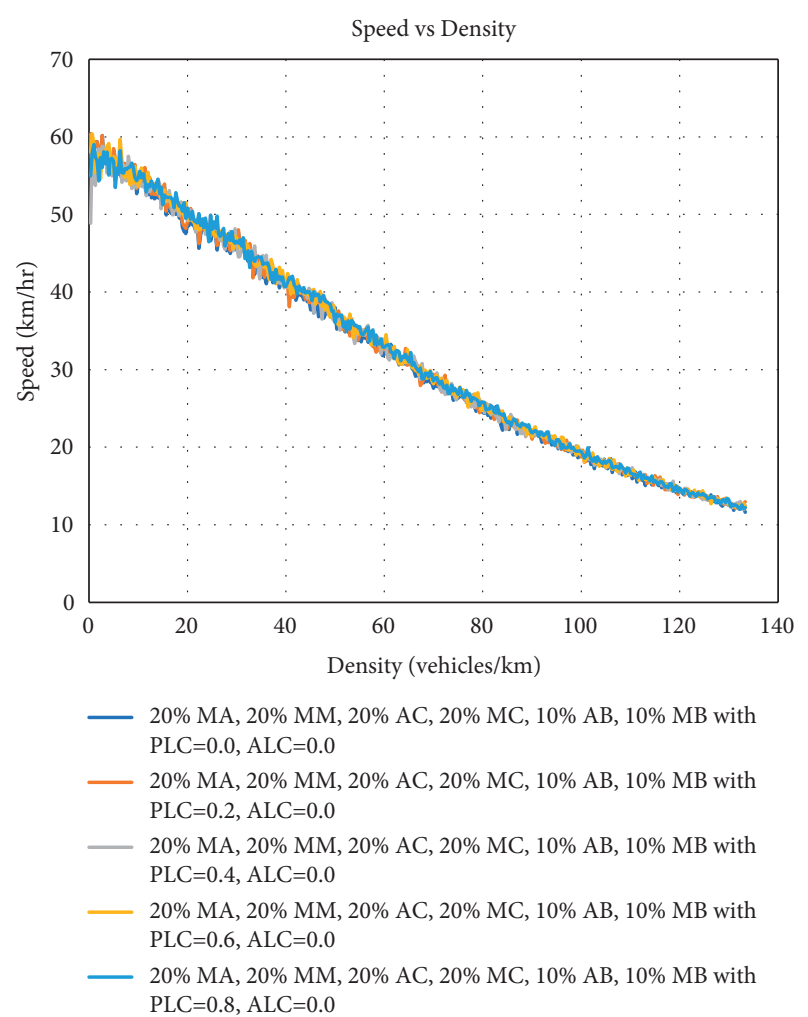

Figure 6: Speed vs. density fundamental diagrams for different compositions of MA, MM, AC, MC, AB, and MB with different values of PLC when ALC is fixed at 0.

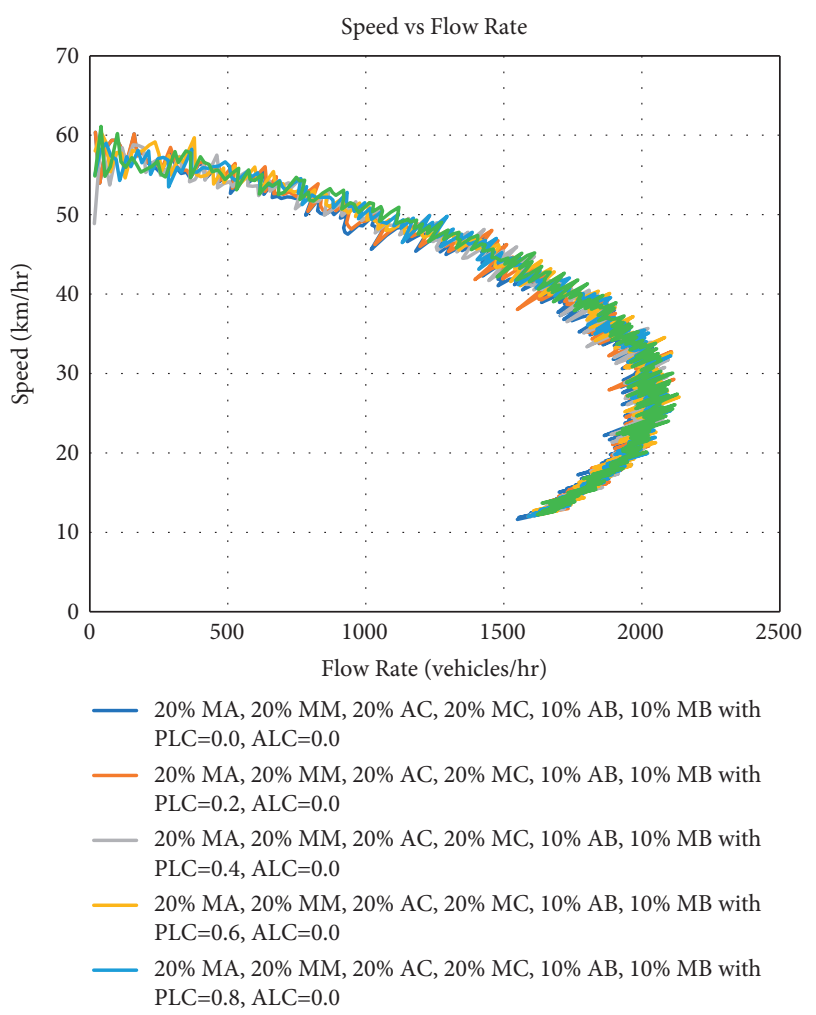

Figure 7: Speed vs. flow rate fundamental diagrams for different compositions of MA, MM, AC, MC, $\mathrm{AB}$, and $\mathrm{MB}$ with different values of PLC when ALC is fixed at 0 .

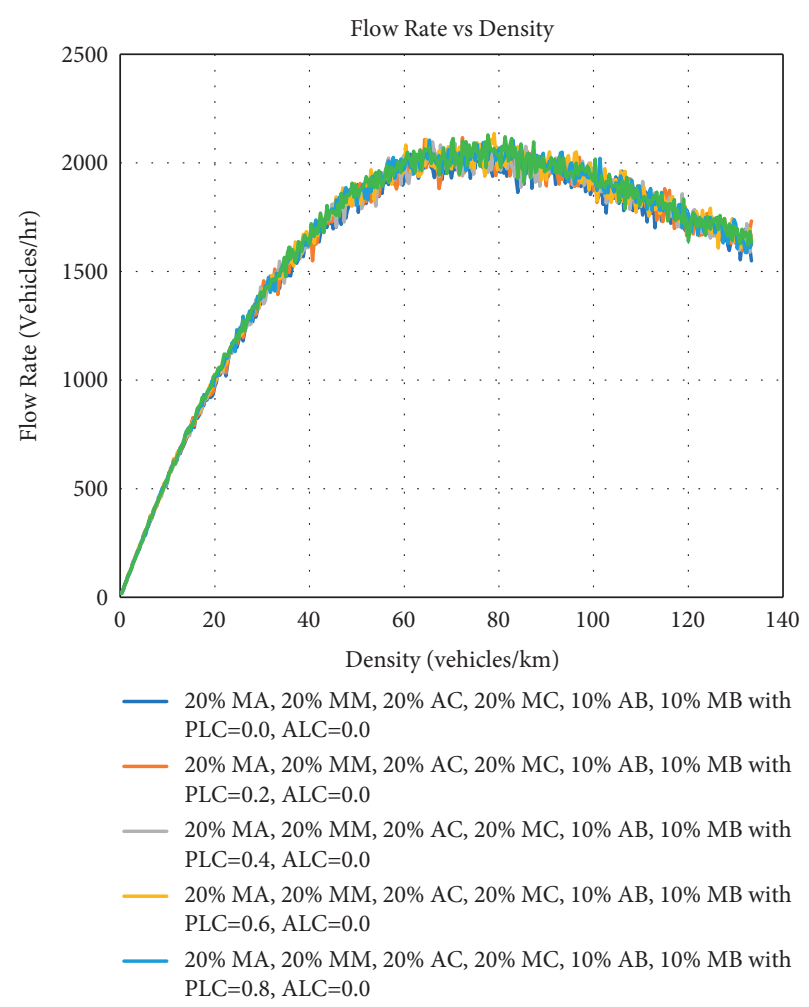

FIgURE 8: Flow rate vs. density fundamental diagrams for different compositions of MA, MM, AC, MC, $\mathrm{AB}$, and $\mathrm{MB}$ with different values of PLC when ALC is fixed at 0.

capacity). Thus, aggressive behavior can impact the capacity of the network in an uprising manner.

9.4. Scenario 4. In this setup, the composition is the following:

8\% MA, 36\% MM, 8\% AC, 36\% MC, $4 \%$ AB, $8 \%$ MB with different values of ALC (0.2 to 0.8$)$ and PLC (0.2 to $0.8)$

Figures, 12-14 present the traffic flow fundamental diagrams of scenario 4 . There are 4 different types of simulations in this scenario with different values of PLC and ALC in each one. The compositions in this scenario are different from previous ones to emphasize the importance of lanechanging behavior in any heterogeneous traffic arrangement. As evident from the figures, as the probability of ALC increases and PLC decreases, the overall flow rate and speed increase under the same density. When PLC is 0.8 and ALC is 0.2 , the flow rate is 2228 vehicles per hour. In comparison, when PLC is 0.2 and ALC is 0.8 , the flow rate is 2425 vehicles per hour (around a 9 percent increase). Thus, this reinforces the finding of previous scenarios as well that aggressive lanechanging behavior increases the capacity of a network more considerably than polite lane change.

9.5. Scenario 5. In this setup, the composition is the following:

$100 \% \mathrm{MA}$ and 100\%MM (PLC and $\mathrm{ALC}=0.5)$ 


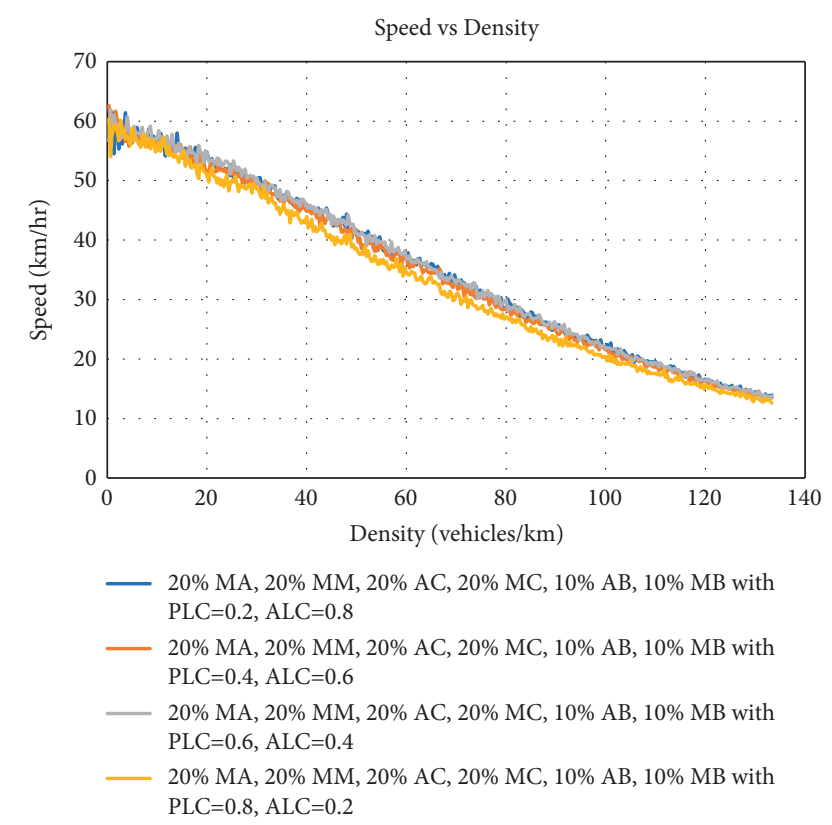

Figure 9: Speed vs. density fundamental diagrams for different compositions of $\mathrm{MA}, \mathrm{MM}, \mathrm{AC}, \mathrm{MC}, \mathrm{AB}$, and $\mathrm{MB}$ with different values of ALC and PLC.

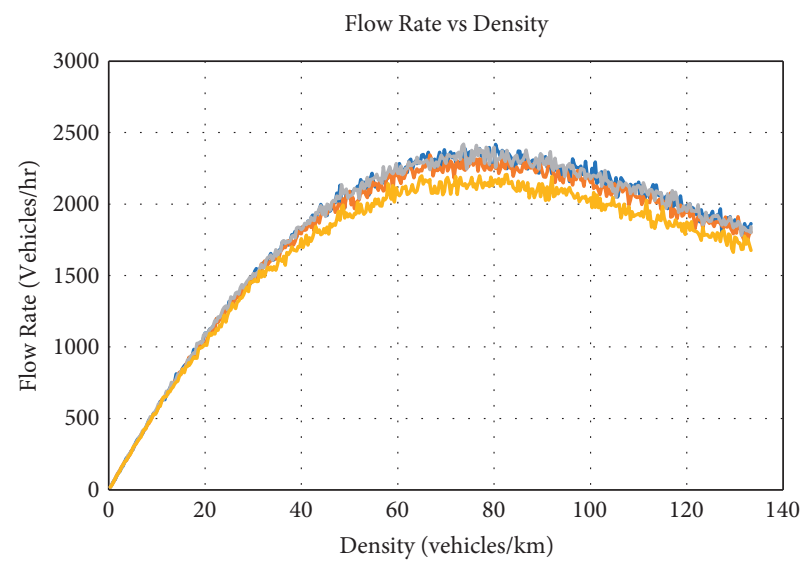

\footnotetext{
$20 \% \mathrm{MA}, 20 \% \mathrm{MM}, 20 \% \mathrm{AC}, 20 \% \mathrm{MC}, 10 \% \mathrm{AB}, 10 \% \mathrm{MB}$ with $\mathrm{PLC}=0.2, \mathrm{ALC}=0.8$

- 20\% MA, 20\% MM, 20\% AC, $20 \%$ MC, $10 \%$ AB, $10 \%$ MB with $\mathrm{PLC}=0.4, \mathrm{ALC}=0.6$

- 20\% MA, $20 \%$ MM, $20 \%$ AC, $20 \%$ MC, $10 \%$ AB, $10 \%$ MB with PLC $=0.6$, ALC $=0.4$

— $20 \% \mathrm{MA}, 20 \% \mathrm{MM}, 20 \% \mathrm{AC}, 20 \% \mathrm{MC}, 10 \% \mathrm{AB}, 10 \% \mathrm{MB}$ with $\mathrm{PLC}=0.8, \mathrm{ALC}=0.2$
}

Figure 10: Flow rate vs. density fundamental diagrams for different compositions of $\mathrm{MA}, \mathrm{MM}, \mathrm{AC}, \mathrm{MC}, \mathrm{AB}$, and $\mathrm{MB}$ with different values of ALC and PLC.

Figures 15-18 present the traffic flow fundamental diagrams of scenario 5 . There are 2 different types of simulations in this scenario with fixed values of PLC and ALC in each one. This simulation aims to show whether micro cars are automated on-road and what kind of impact can they have on the overall capacity and speed of the network. As it is apparent from the above figures,

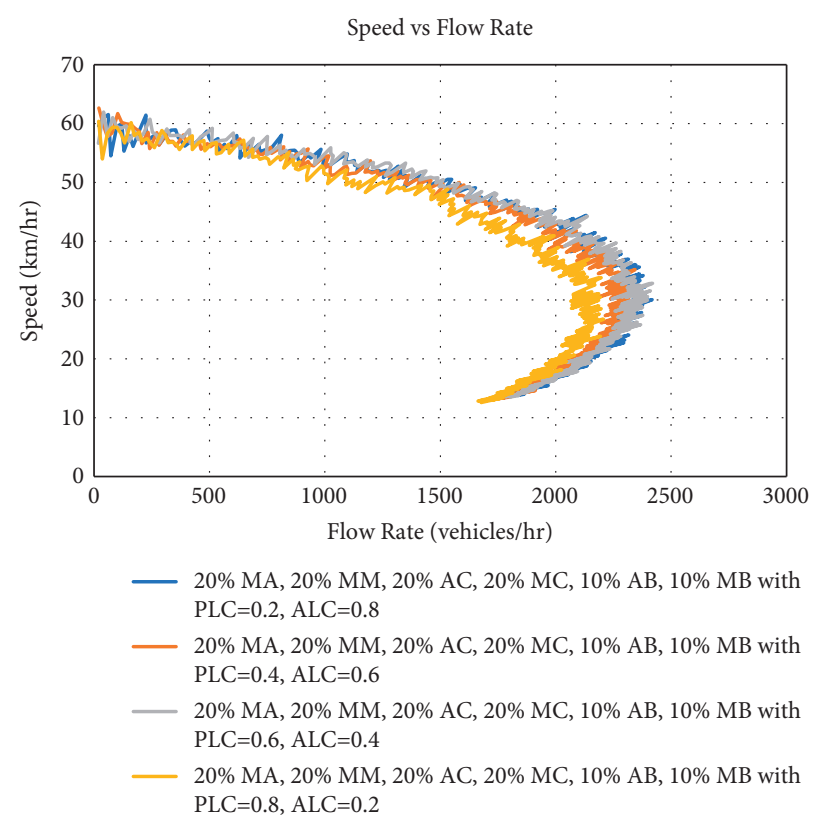

FIgURE 11: Speed vs. flow rate fundamental diagrams for different compositions of $\mathrm{MA}, \mathrm{MM}, \mathrm{AC}, \mathrm{MC}, \mathrm{AB}$, and $\mathrm{MB}$ with different values of ALC and PLC.

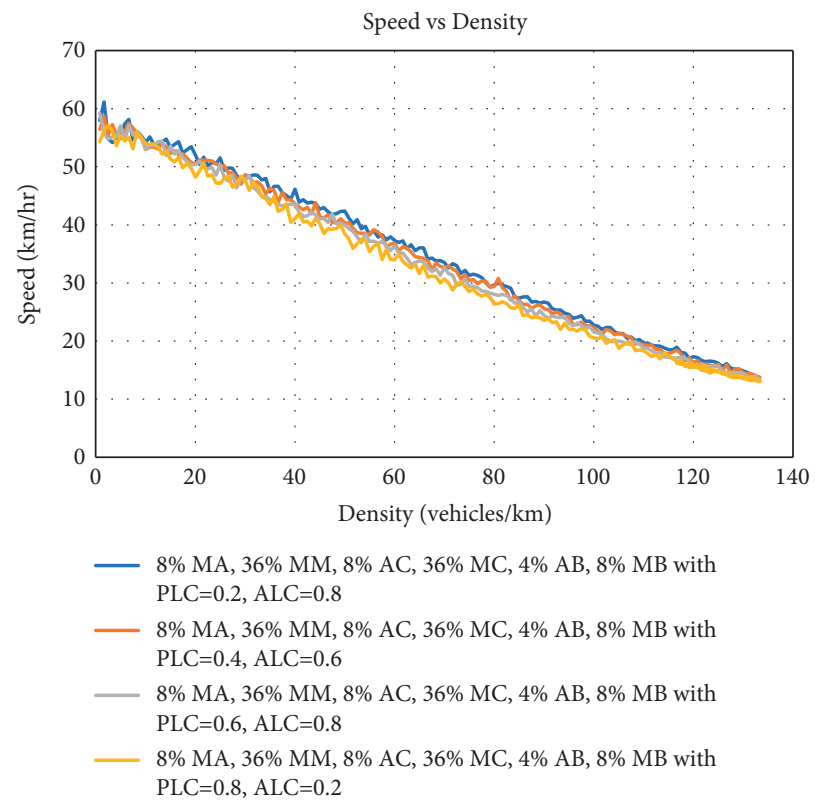

FIGURE 12: Speed vs. density fundamental diagrams for different compositions of MA, MM, AC, MC, $\mathrm{AB}$, and $\mathrm{MB}$ with different values of ALC and PLC.

autonomous micro cars exceed manual micro cars in terms of capacity. As soon as the no. of vehicles increases, from 500 onwards, autonomous micro cars perform efficiently due to their less reaction delay. When the vehicles exceed 2500 and are entering into the congested phase, the flow rate of both vehicles in comparison starts to come near one another but the difference still remains. This is due to the fact the density is also increasing constantly with increasing 

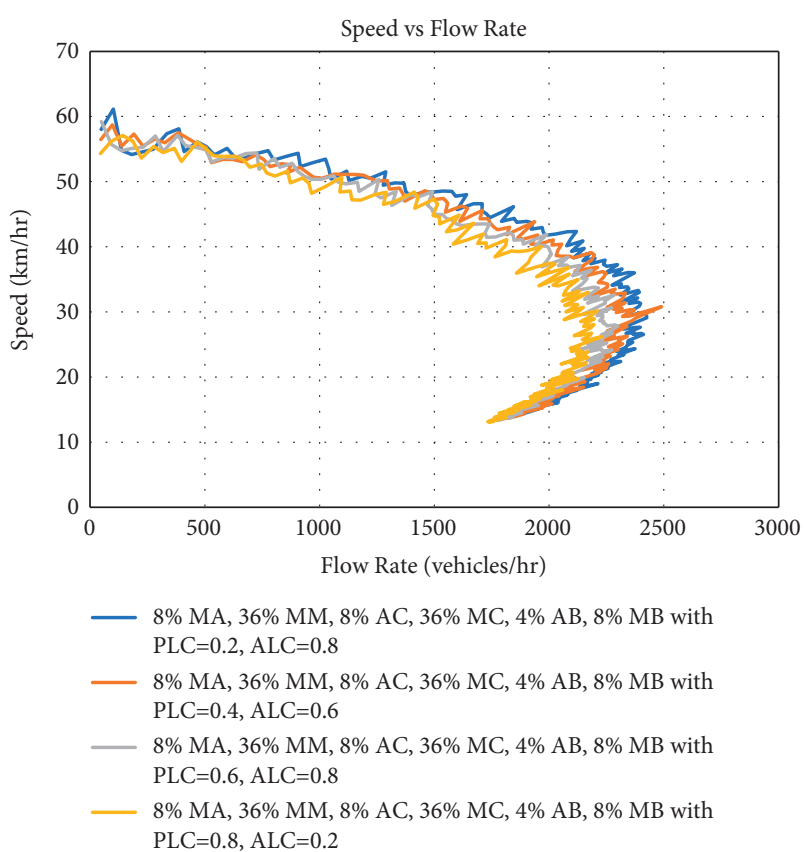

FIgURE 13: Speed vs. flow rate fundamental diagrams for different compositions of MA, MM, AC, MC, AB, and $\mathrm{MB}$ with different values of ALC and PLC.

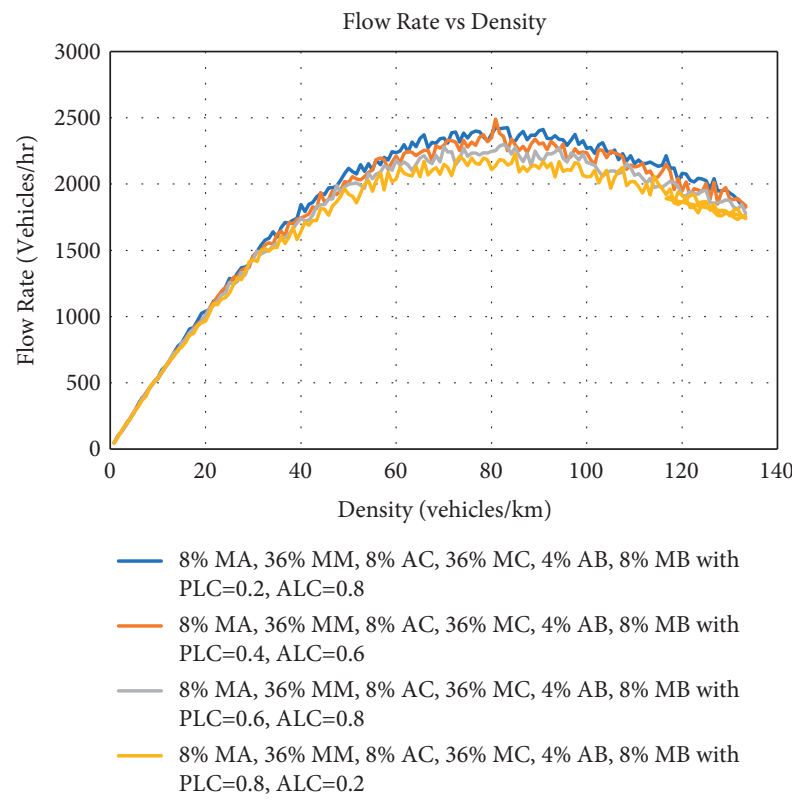

FIGURE 14: Flow rate vs. density fundamental diagrams for different compositions of MA, MM, AC, MC, $\mathrm{AB}$, and $\mathrm{MB}$ with different values of ALC and PLC.

no. of vehicles. The maximum flow rate of manual micro cars is 4200 vehicles per hour and the maximum flow rate of autonomous micro cars is 4455 (around a 6 percent increase in capacity). A similar pattern is seen for average speed. The average speed of autonomous micro cars is

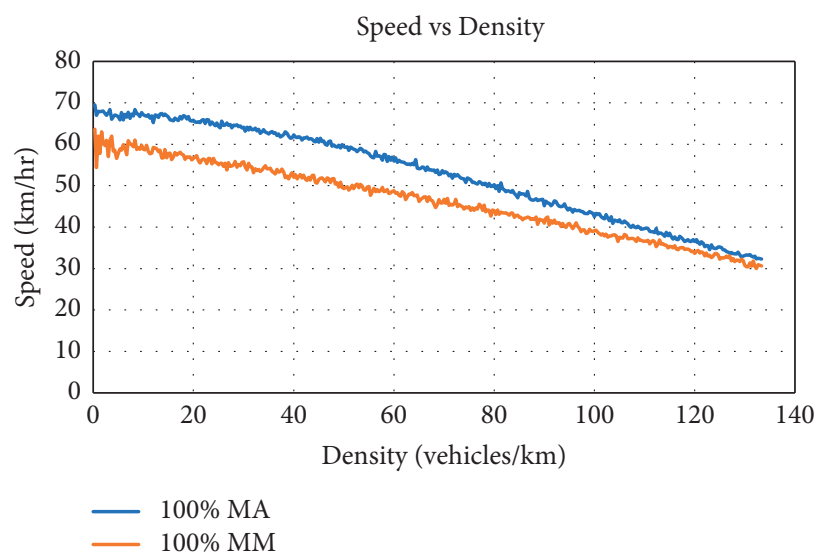

FIgURE 15: Speed vs. density fundamental diagrams for different compositions of 100 percent MA vs. 100 percent MM.

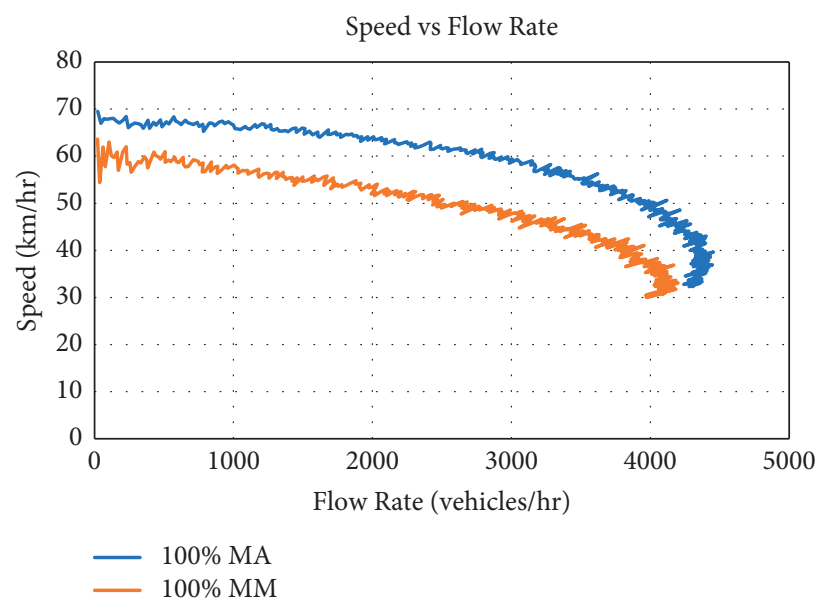

FIgURE 16: Speed vs. flow rate fundamental diagrams for different compositions of 100 percent MA vs. 100 percent MM.

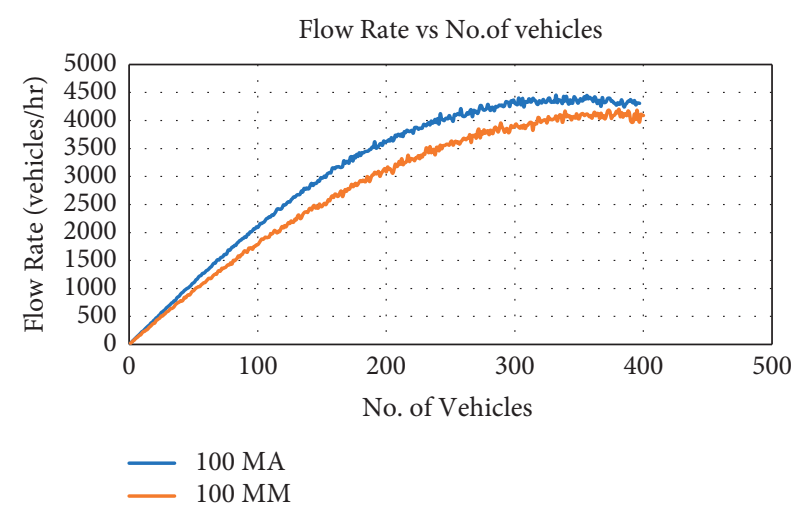

FIgURE 17: Flow rate vs. no. of vehicles fundamental diagrams for different compositions of 100 percent MA vs. 100 percent MM.

higher throughout the simulation than manual micro cars and only comes close to one another when the density becomes too high. 


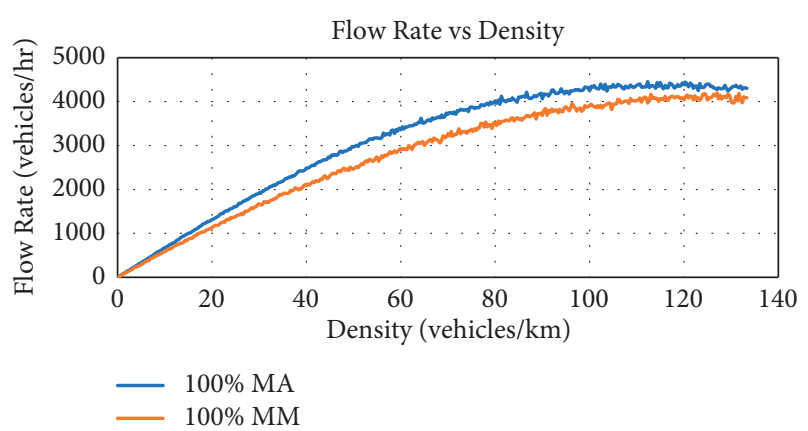

FIGURE 18: Flow rate vs. density fundamental diagrams for different compositions of 100 percent MA vs. 100 percent MM.

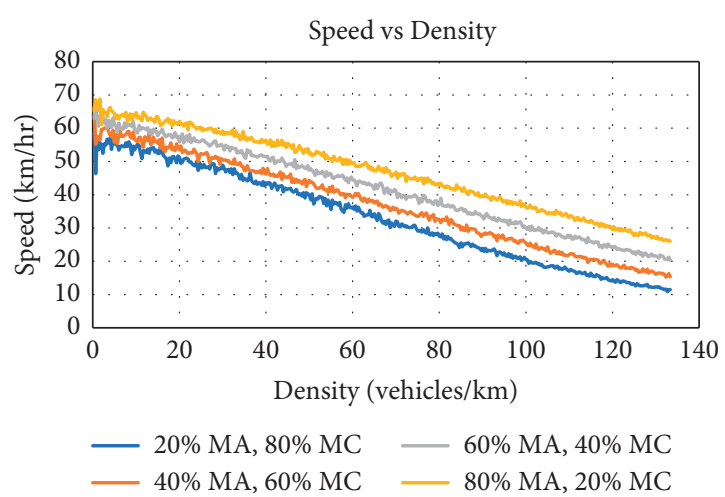

FIGURE 19: Speed vs. density fundamental diagrams for different compositions of MA and MC.

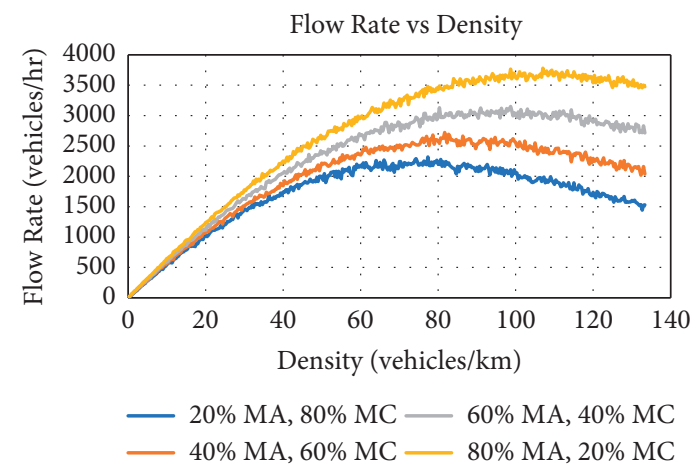

FIGURE 20: Flow rate vs. density fundamental diagrams for different compositions of MA and MC.

9.6. Scenario 6. In this setup, the composition is the following:

$$
\begin{aligned}
& 20 \% \mathrm{MA}+80 \% \mathrm{MC}, \quad 40 \% \quad \mathrm{MA}+60 \% \mathrm{MC}, \quad 60 \% \\
& \mathrm{MA}+40 \% \mathrm{MC}, \quad 80 \% \quad \mathrm{MA}+20 \% \mathrm{MC} \quad \text { (PLC and } \\
& \mathrm{ALC}=0.5 \text { ) }
\end{aligned}
$$

Figures 19-22 present the traffic flow fundamental diagrams of scenario 6 . There are 4 different types of simulations in this scenario with fixed values of PLC and ALC in each one. The goal of this scenario is to assess the performance of a network, in which autonomous micro cars run

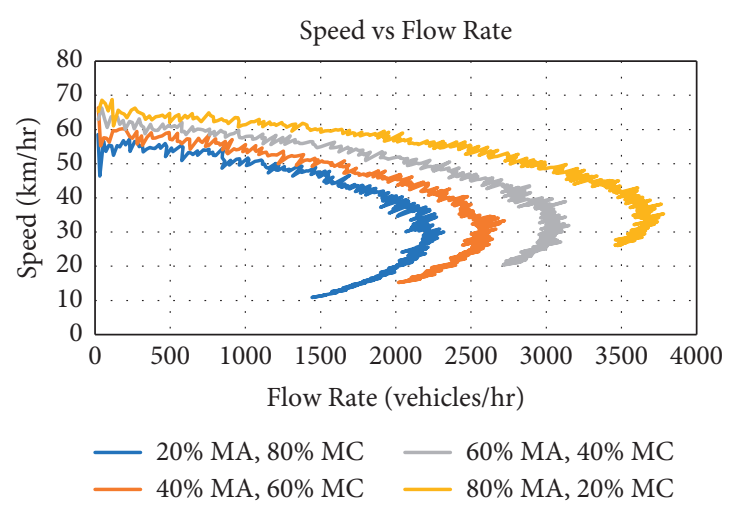

FIgURE 21: Speed vs. flow rate fundamental diagrams for different compositions of MA and MC.

parallel with manual cars. The impact of Autonomous micro cars in the overall network can be seen evidently in the figures as soon as the no. of total vehicles exceeds 500. Since autonomous micro cars have less reaction delay, they can perform more efficiently. Similarly, since their size is less in comparison to normal cars, the increase in flow rate is significant when the percentage of micro autonomous cars is $80 \%$ and manual cars is $20 \%$. As the penetration rate of autonomous micro cars increases, the average speed of the network also increases. This means that more vehicles can change lane efficiently, and the speed of autonomous micro cars is greater than manual cars if their percentage is high, which subsequently increases the network's average speed. When the percentage of autonomous micro cars is $20 \%$, and the percentage of manual cars is $80 \%$, the maximum flow rate is 2310 vehicles per hour. In comparison, when the percentage of autonomous micro cars is $80 \%$, and the percentage of manual cars is $20 \%$, the maximum flow rate is 3780 vehicles per hour ( $63 \%$ increase in capacity). Thus, the autonomous micro cars can increase the flow rate significantly if deputed in high volume.

9.7. Scenario 7. In this setup, the composition is the following:

$20 \% \mathrm{MA}+80 \% \mathrm{AC}, 40 \% \mathrm{MA}+60 \% \mathrm{AC}, 60 \% \mathrm{MA}+40 \%$ $\mathrm{AC}, 80 \% \mathrm{MA}+20 \% \mathrm{AC}(\mathrm{PLC}$ and $\mathrm{ALC}=0.5)$

Figures 23-26 present the traffic flow fundamental diagrams of scenario 7 . There are 4 different types of simulations in this scenario with fixed values of PLC and ALC in each one. The goal of this scenario is to assess the performance of a network in which autonomous micro cars run parallel with autonomous cars. In the free-flow phase, all 4 types of simulations perform similarly but when they approach the congestion phase; the superiority of autonomous micro cars due to their smaller size can be clearly observed. Since their size is less and reaction delay is similar to autonomous cars, when density becomes high, they can change lane efficiency more easily than autonomous cars. Thus, the capacity and average speed of network increase. For example, when the percentage of autonomous micro cars is $20 \%$ and the percentage of autonomous cars is $80 \%$, the 
maximum flow rate is 3500 vehicles/hr. In comparison, when the percentage of autonomous micro cars is $80 \%$, and the percentage of autonomous cars is $20 \%$, the maximum flow rate is 4236 vehicles/ $\mathrm{hr}$ ( $21 \%$ increase in capacity). Thus, micro autonomous cars, when run parallel with autonomous cars, can increase the capacity significantly than only autonomous cars.

Furthermore, the average vehicle occupancy is 1.7 in the US (Federal Highway Administration 2018) and micro car can easily carry 2 passengers. If 1000 micro autonomous cars are traveling on road, then 1700 passengers can be easily accommodated compared to 1000 autonomous cars traveling on another road carrying the same no. of passengers. Since autonomous micro cars will be less in size, they can easily change lanes and significantly increase the network's capacity, resulting in fewer delays and reduced congestion compared to using only autonomous cars. This is one of the key benefits of using the autonomous micro cars on roads in parallel with autonomous cars which is also highlighted in outputs of scenario 8 .

9.8. Scenario 8. In this setup, the composition is the following:

$$
\begin{aligned}
& 20 \% \quad \mathrm{MA}+80 \% \mathrm{MM}, \quad 40 \% \quad \mathrm{MA}+60 \% \mathrm{MM}, \quad 60 \% \\
& \mathrm{MA}+40 \% \mathrm{MM}, \quad 80 \% \quad \mathrm{MA}+20 \% \mathrm{MM} \quad \text { (PLC and } \\
& \mathrm{ALC}=0.5 \text { ) }
\end{aligned}
$$

Figures 27-30 present the traffic flow fundamental diagrams of scenario 8 . There are 4 different types of simulations in this scenario with fixed values of PLC and ALC in each one. The goal of this scenario is to assess the performance of a network in which autonomous micro cars run parallel with manual micro cars. In the start, all 4 types of simulations perform similarly but when the numbers of vehicles increases, the superiority of autonomous micro cars due to their less reaction delay can be visibly observed. The high penetration rate of autonomous micro cars results in more capacity and an increase in average speed in the network. When the percentage of autonomous micro cars is $20 \%$ with $80 \%$ of manual micro cars, the maximum flow rate is 4231 vehicles/hr. in comparison, when the percentage of autonomous micro cars is $80 \%$ with $20 \%$ of manual micro cars, the maximum flow rate is 4372 vehicles/hr (around a $3 \%$ increase). This means that if only micro cars run on the network, capacity can be significantly increased, especially if average passenger vehicle occupancy is less than 2 .

9.9. Scenario 9. In this setup, the composition is the following:

$$
\begin{aligned}
& 20 \% \mathrm{MM}+80 \% \mathrm{AC}, 40 \% \mathrm{MM}+60 \% \mathrm{AC}, 60 \% \mathrm{MM}+40 \% \\
& \mathrm{AC}, 80 \% \mathrm{MM}+20 \% \mathrm{AC}(\mathrm{PLC} \text { and } \mathrm{ALC}=0.5)
\end{aligned}
$$

Figures 31-34 present the traffic flow fundamental diagrams of scenario 9. There are four different types of simulations in this scenario with fixed values of PLC and ALC in each one. The goal of this scenario is to assess the performance of a network in which manual micro cars run parallel with autonomous cars. In the free-flow phase, all 4

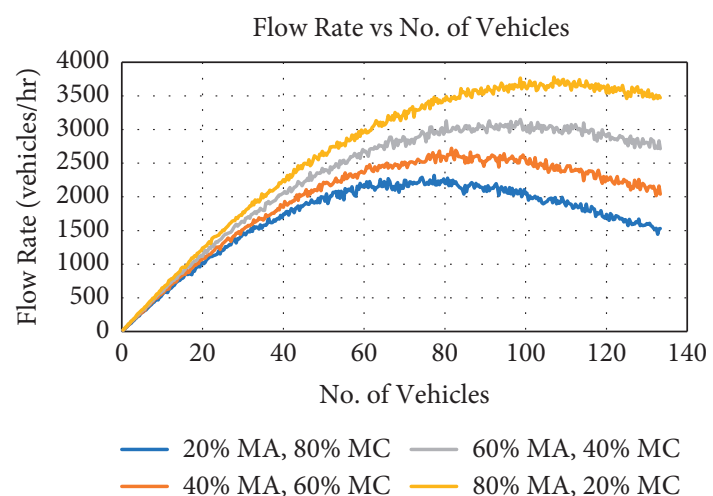

FIgURE 22: Flow rate vs. no. of vehicles fundamental diagrams for different compositions of MA and MC.

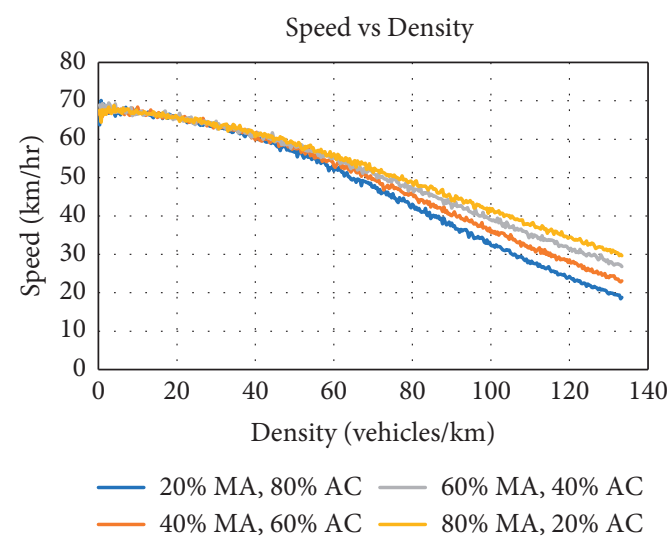

FIgURE 23: Speed vs. density fundamental diagrams for different compositions of MA and AC.

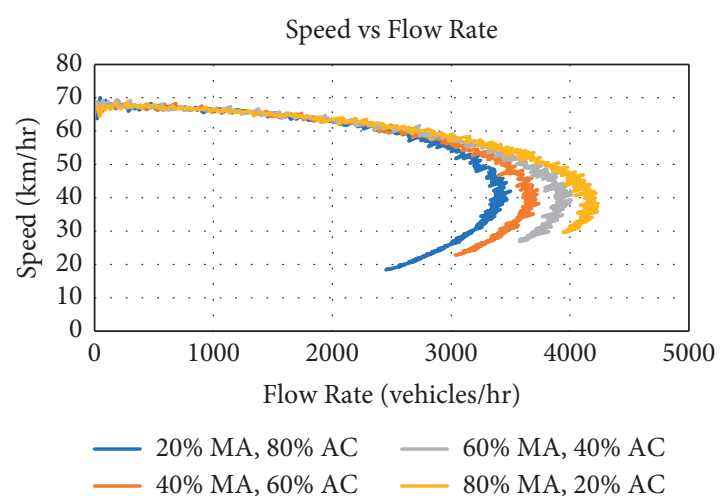

FIgURE 24: Speed vs. flow rate fundamental diagrams for different compositions of MA and AC.

types of simulations perform similarly but when they approach the congestion phase, the superiority of high penetration rate of manual micro cars due to their smaller size can be clearly observed. The main finding here is that when the percentage of manual micro cars was $80 \%$ and autonomous cars was $20 \%$, the flow rate was less than other simulations. When the total no. of vehicles exceeds 2200 vehicles, the high penetration of micro manual cars increases 


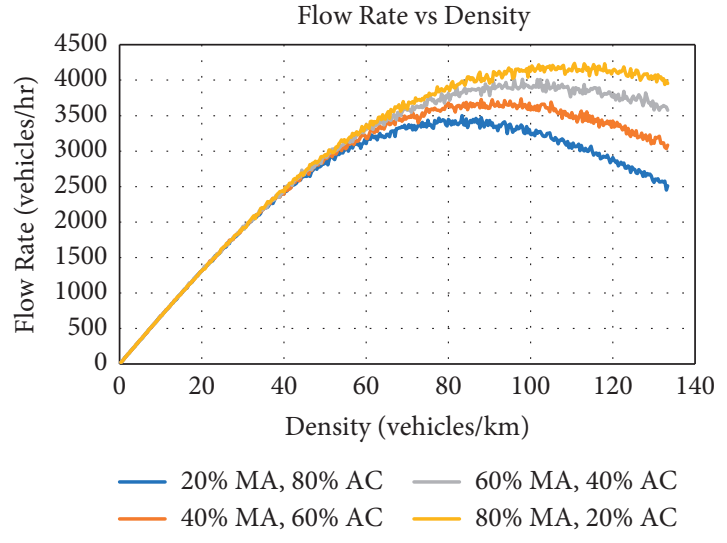

FIGURE 25: Flow rate vs. density fundamental diagrams for different compositions of MA and AC.

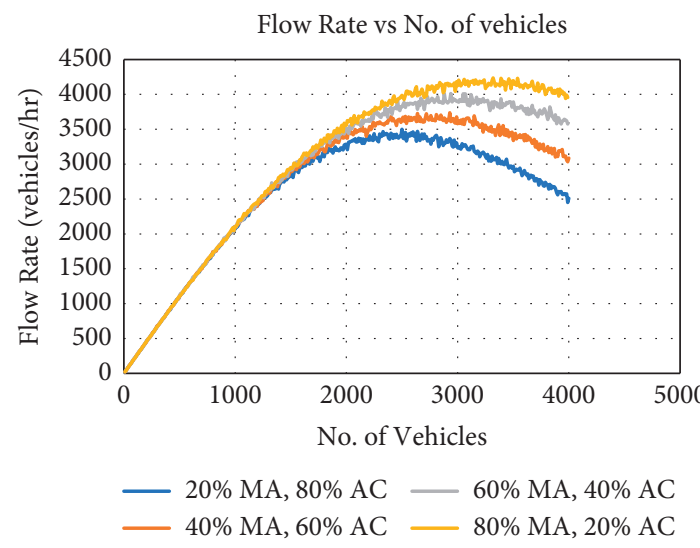

FIGURE 26: Flow rate vs. no. of vehicles fundamental diagrams for different compositions of MA and AC.

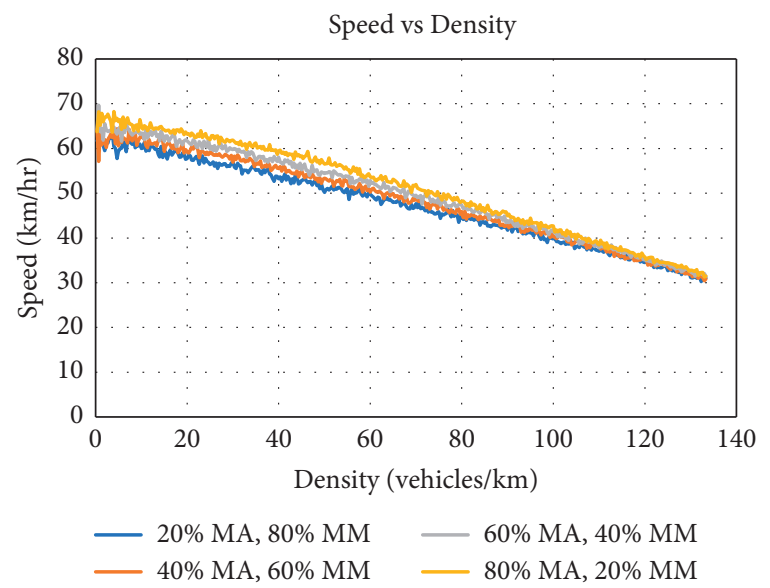

Figure 27: Speed vs. density fundamental diagrams for different compositions of MA and MM.

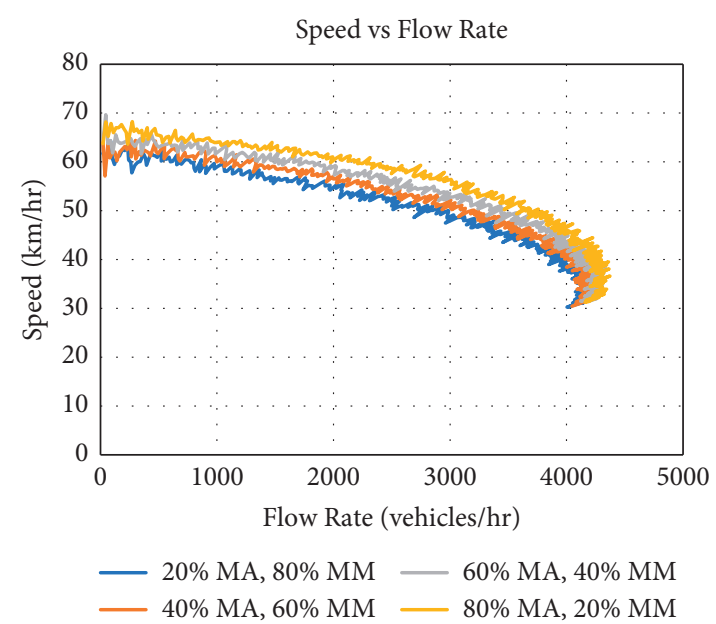

FIgURE 28: Speed vs. flow rate fundamental diagrams for different compositions of MA and MM.

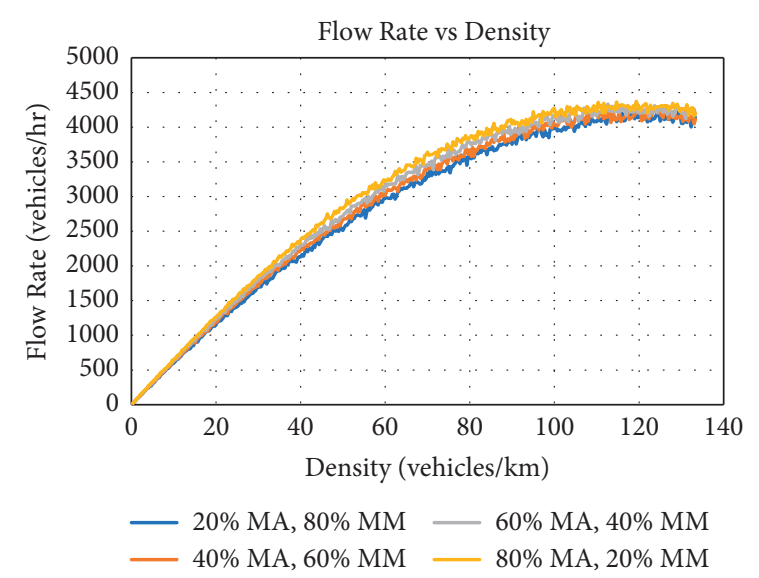

FIGURE 29: Flow rate vs. density fundamental diagrams for different compositions of MA and MM.

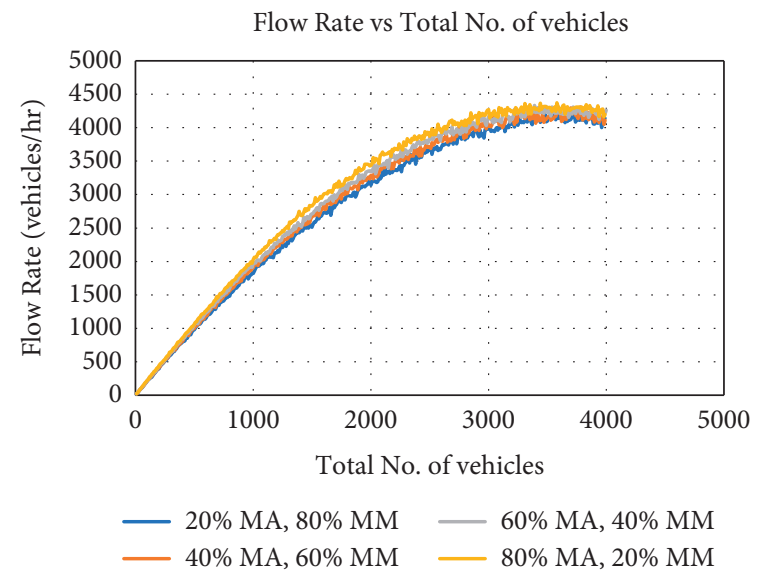

FIgURE 30: Flow rate vs. no. of vehicles fundamental diagrams for different compositions of MA and MM. 


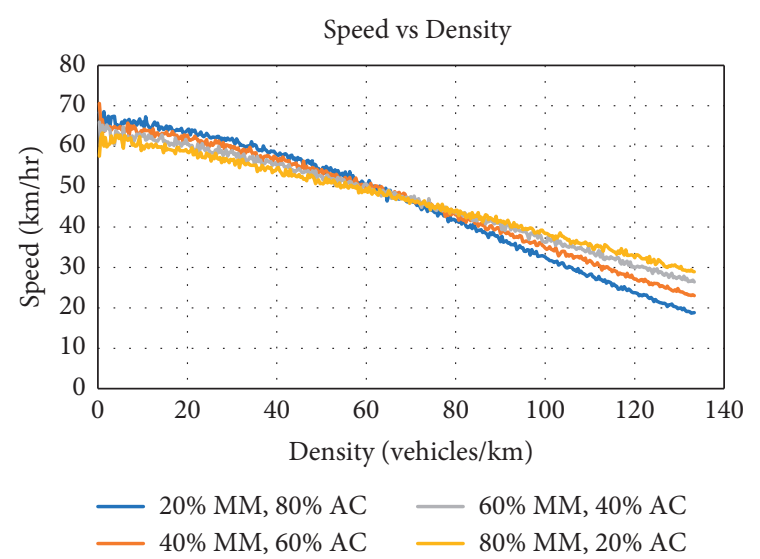

Figure 31: Speed vs. density fundamental diagrams for different compositions of MM and AC.

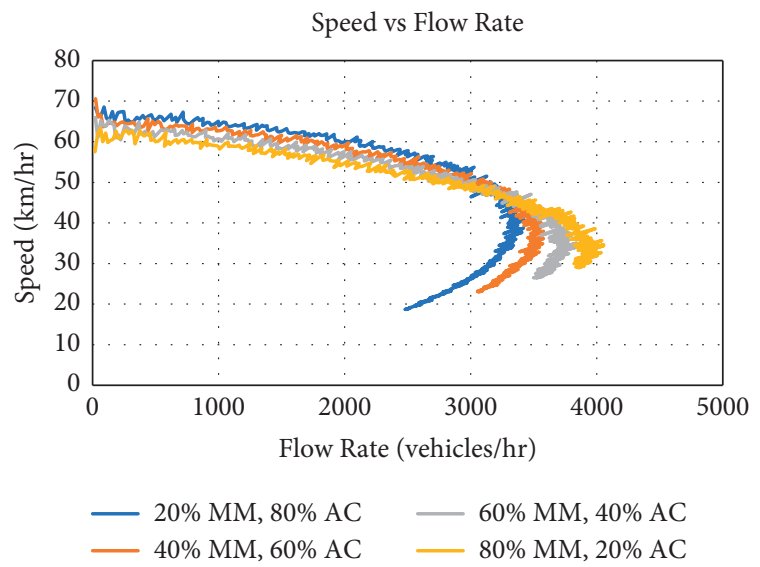

Figure 32: Speed vs. flow rate fundamental diagrams for different compositions of $\mathrm{MM}$ and $\mathrm{AC}$.

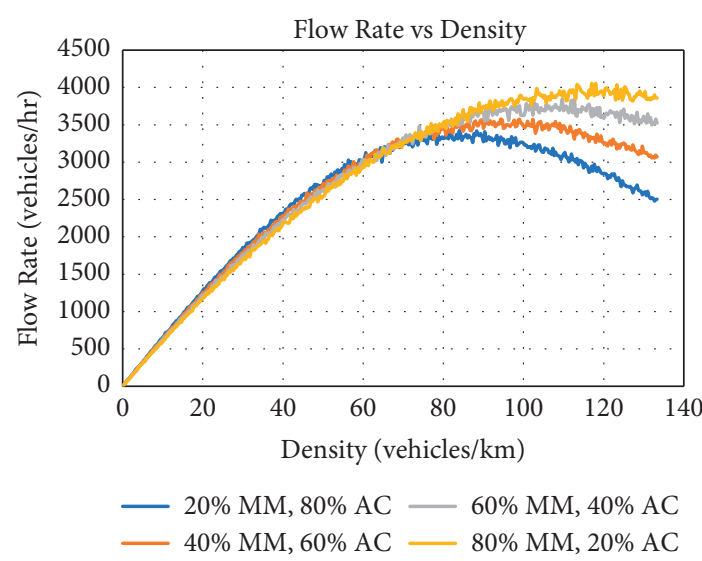

FIGURE 33: Flow rate vs. density fundamental diagrams for different compositions of MM and AC.

the flow rate and average speed. Furthermore, the difference in flow rate with other simulations becomes high with increasing no. of vehicles. This is because of their smaller size;

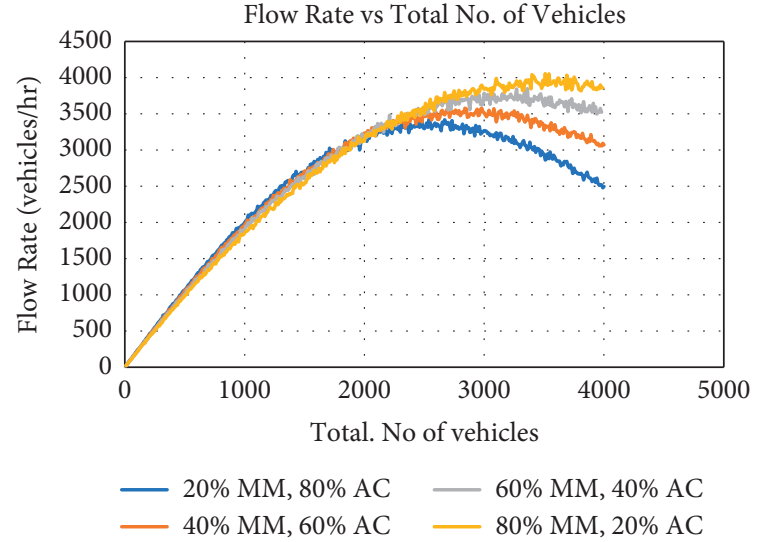

Figure 34: Flow rate vs. total no. of vehicles fundamental diagrams for different compositions of MM and AC.

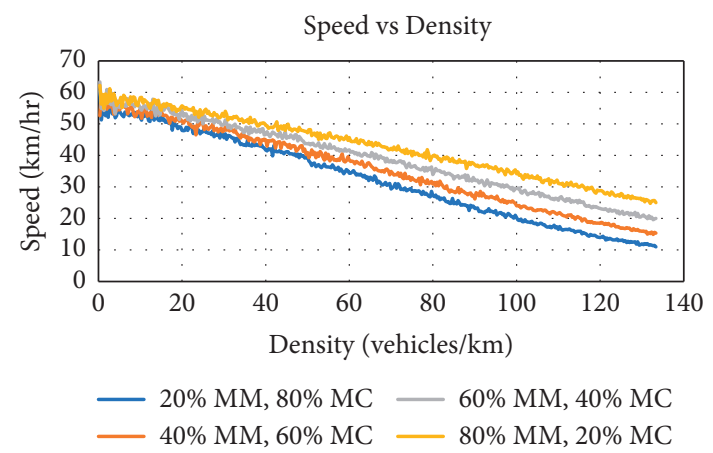

FIgURe 35: Flow rate vs. density fundamental diagrams for different compositions of $\mathrm{MM}$ and $\mathrm{MC}$.

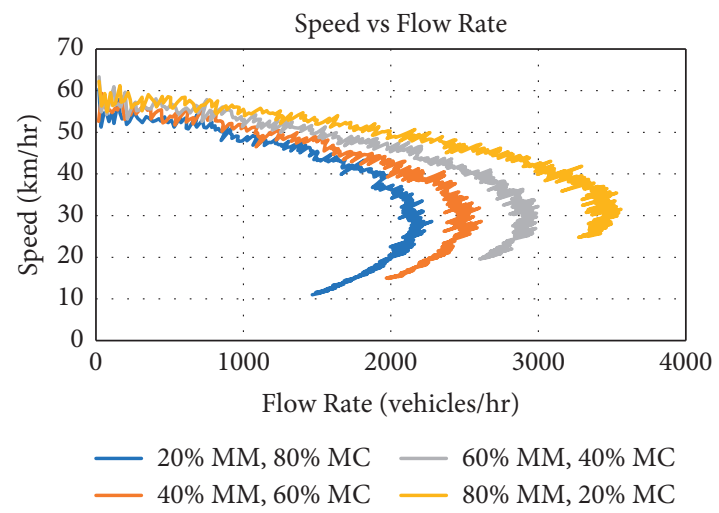

FIgURE 36: Speed vs. flow rate fundamental diagrams for different compositions of MM and MC.

manual micro cars can change lane efficiently. When the percentage of manual micro cars was $20 \%$ and autonomous cars was $80 \%$, the maximum flow rate was 3424 vehicles $/ \mathrm{hr}$. In comparison, when the percentage of manual micro cars was $80 \%$ and autonomous cars was $20 \%$, the maximum flow rate became 4055 vehicles/hr (around an 18\% increase). Thus, similar to autonomous micro cars, a high percentage 


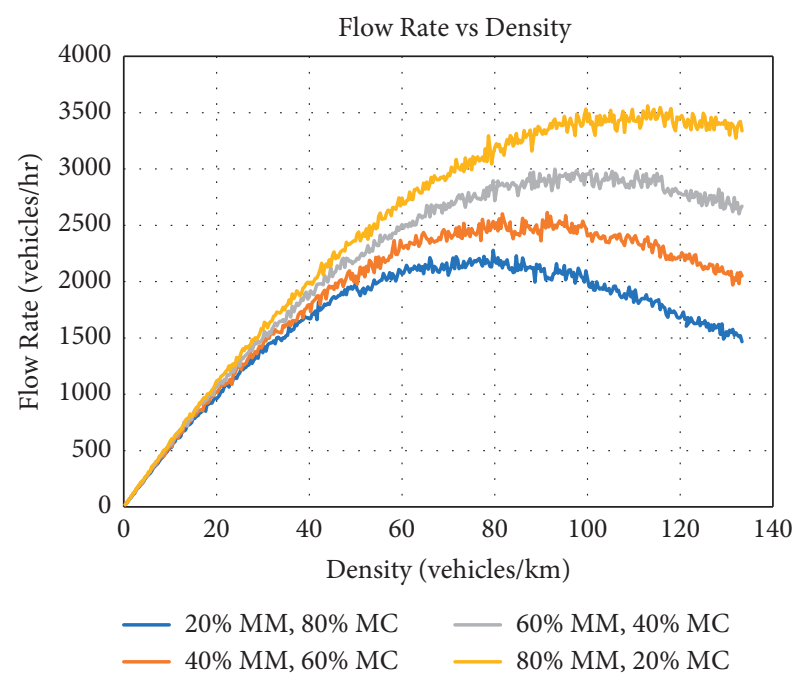

FIGURE 37: Flow rate vs. density fundamental diagrams for different compositions of MM and MC.

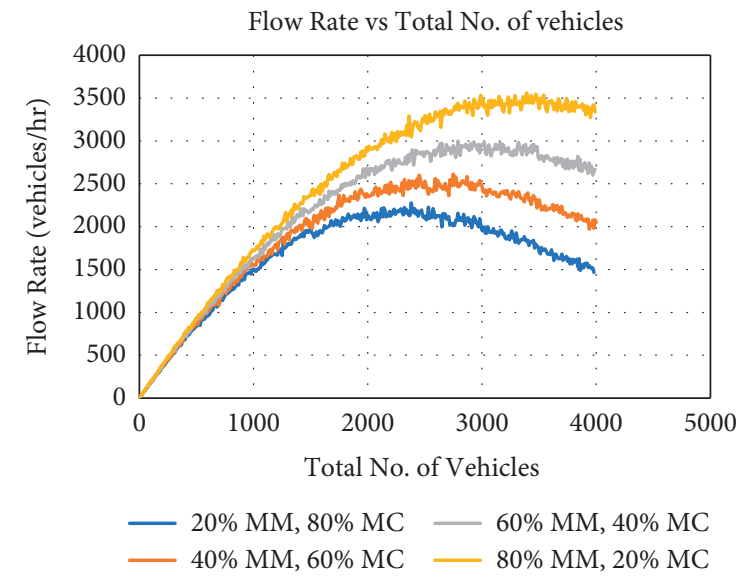

FIGURE 38: Flow rate vs. total no. of vehicles fundamental diagrams for different compositions of MM and MC.

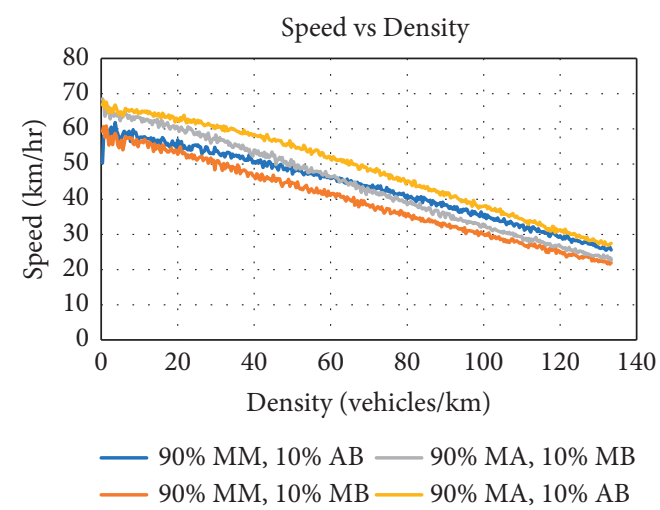

Figure 39: Speed vs. density fundamental diagrams for different compositions of $\mathrm{MA}, \mathrm{MM}, \mathrm{AB}$, and $\mathrm{MB}$.

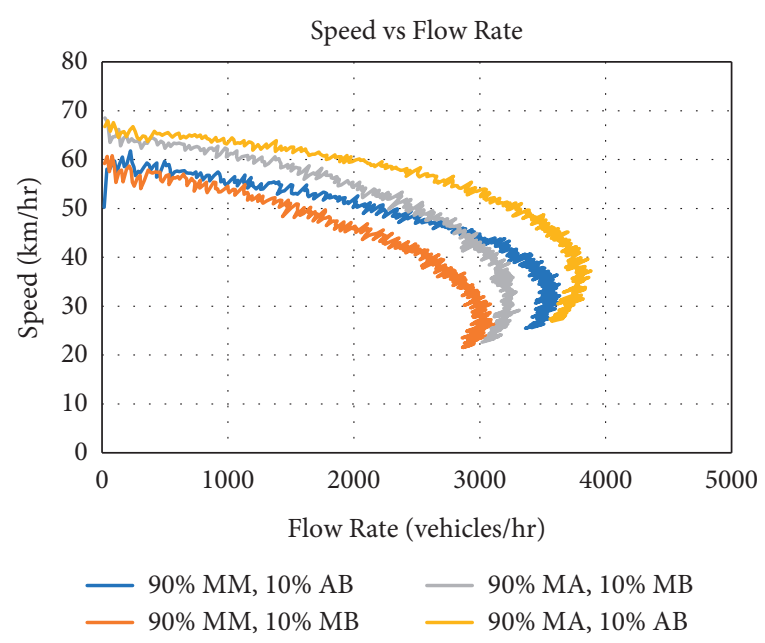

FIgUre 40: Speed vs. flow rate fundamental diagrams for different compositions of $\mathrm{MA}, \mathrm{MM}, \mathrm{AB}$, and $\mathrm{MB}$.

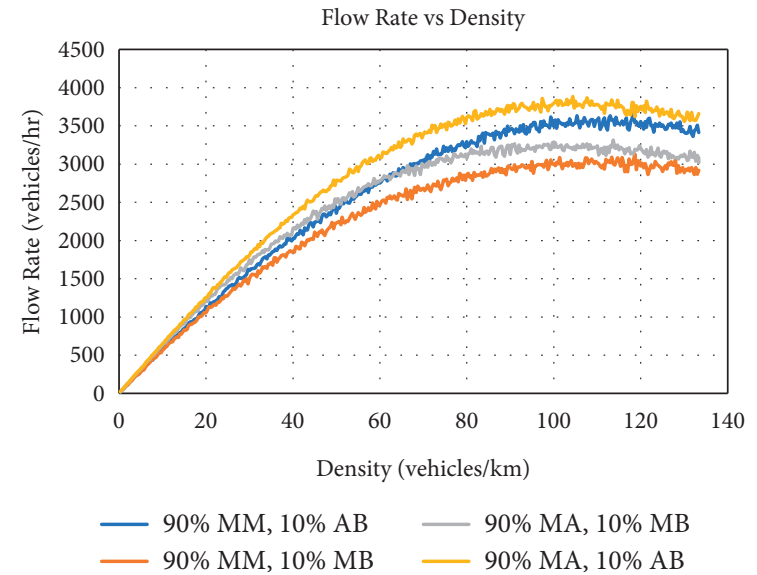

FIgURE 41: Flow rate vs. density fundamental diagrams for different compositions of $\mathrm{MA}, \mathrm{MM}, \mathrm{AB}$, and $\mathrm{MB}$.

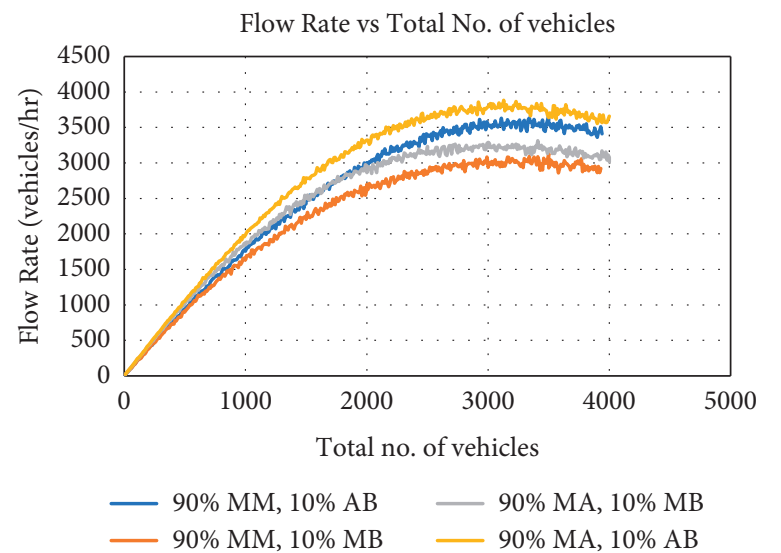

FIgURE 42: Flow rate vs. total no. of vehicles fundamental diagrams for different compositions of $\mathrm{MA}, \mathrm{MM}, \mathrm{AB}$, and $\mathrm{MB}$. 
TABle 4: Average vehicle occupancy of cars in different countries.

\begin{tabular}{llc}
\hline Country & Year & Average vehicle occupancy of cars (number of persons/car) \\
\hline USA (FHWA 2018) [35] & 2017 & 1.7 \\
England (England 2002 to 2018) [36] & 2018 & 1.6 \\
Australia (ABS 2016) [37] & 2014 & 1.57 \\
India [38] & 2006 & 2.5 \\
Canada (CPA 2009) [39] & 2009 & 1.62 \\
European countries (CWAI 2016) [40] & 2014 & 1.48 \\
China (Beijing) & 2019 & 1.4 \\
Pakistan (Lahore) & 2019 & 2.2 \\
\hline
\end{tabular}

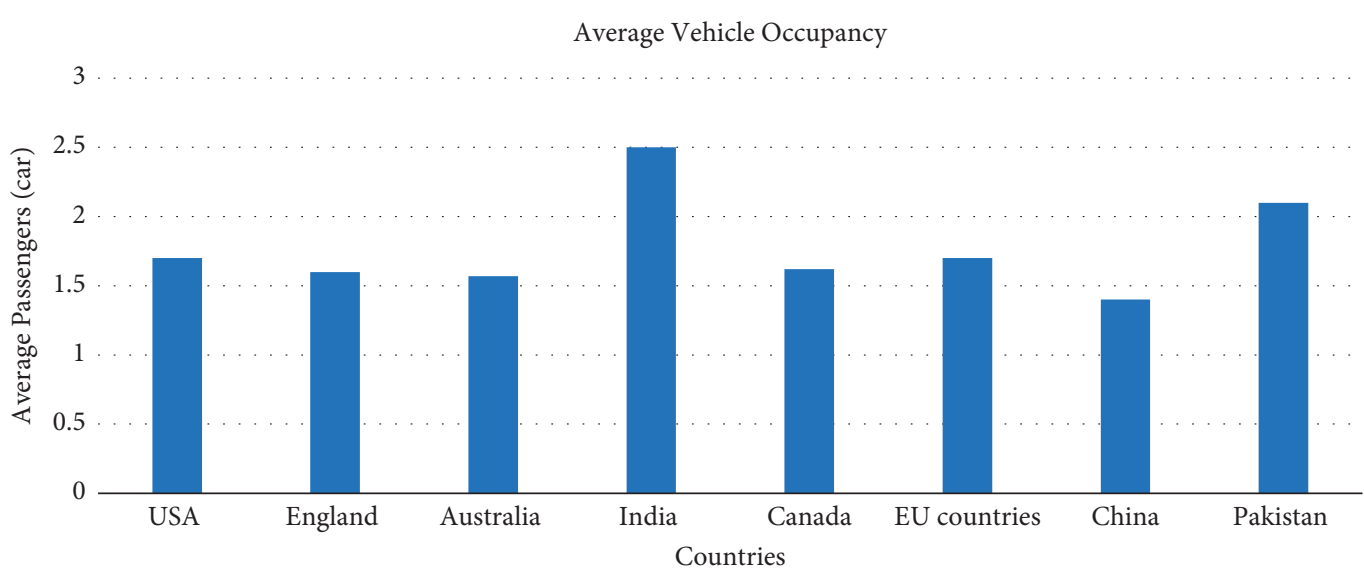

FIGURE 43: Average vehicle occupancy of cars in different countries.

of manual micro cars can also significantly increase the network's capacity if deployed parallelly along with autonomous cars. Where average vehicle occupancy is less than 2 , manual micro cars as per the simulation results greatly reduce traffic congestions and traffic delays.

9.10. Scenario 10. In this setup, the composition is the following:

$$
\begin{aligned}
& 20 \% \mathrm{MM}+80 \% \mathrm{MC}, \quad 40 \% \mathrm{MM}+60 \% \mathrm{MC}, \\
& \mathrm{MM}+40 \% \mathrm{MC}, \quad 80 \% \mathrm{MM}+20 \% \mathrm{MC} \quad(\mathrm{PLC}
\end{aligned}
$$

Figures 35-38 present the traffic flow fundamental diagrams of scenario 10 . There are 4 different types of simulations in this scenario with fixed values of PLC and ALC in each one. The goal of this scenario is to assess the performance of a road segment in which manual micro cars run parallel with manual cars. It is evident from the figures that when the total no. of vehicles is less than 1000, the flow rate of all simulations is near one another but when the no. of vehicles increases, the flow rate rises significantly with increasing no of vehicles in simulation where percentage of manual micro cars is $80 \%$ and manual cars is $20 \%$. When the percentage of manual micro cars is $20 \%$ and that of manual cars is $80 \%$, the maximum flow rate is 2277 vehicles/hr. When the percentage of manual micro cars is $80 \%$ and that of manual cars is $20 \%$, the maximum flow rate is 3560 vehicles/hr (56\% increase in capacity). This further reinforces the finding that micro cars can increase the capacity of a road significantly, reduce traffic congestions and delay, and easily change lanes due to their smaller size. Furthermore, with average vehicle occupancy of less than 2, micro cars can be utilized to improve the existing traffic conditions on any road.

9.11. Scenario 11. In this setup, the composition is the following:

$$
90 \% \mathrm{MM}+10 \% \mathrm{AB}, 90 \% \mathrm{MM}+10 \% \mathrm{MB}, 90 \% \mathrm{MA}+10 \%
$$$$
\mathrm{AB}, 90 \% \mathrm{MA}+10 \% \mathrm{AB}(\mathrm{PLC} \text { and } \mathrm{ALC}=0.5)
$$

Figures 39-42 present the traffic flow fundamental diagrams of scenario 11 . There are 4 different types of simulations in this scenario with fixed values of PLC and ALC in each one. The goal of this scenario is to assess the performance of a segment in which manual and autonomous micro cars run parallel with Manual and autonomous buses. It is evident from the figure that the composition of $90 \% \mathrm{MA}$ and $10 \% \mathrm{AB}$ has the highest flow rate ( 3883 vehicles/hr), almost 23 percent more than when micro manual cars are 90 percent and manual buses are 10 percent. The interesting point here is that when micro manual cars are $90 \%$ and $10 \% \mathrm{AB}$, in congestion phase this composition is performing better than $90 \%$ micro autonomous cars and $10 \% \mathrm{MB}$. This is because, in the congestion phase, autonomous buses can perform much better when coupled with micro cars on roads.

Table 4 and Figure 43 depict the average no of passengers/cars in different countries of the globe. One of the goals of this comparison is to highlight the importance of 
introducing the micro version of autonomous cars into the traffic flow. For instance, all the developed countries have an average vehicle occupancy of less than 2. Alongside the automation technology, the smaller cars will be immensely helpful in abating the traffic congestions on a regional level. Vehicle occupancy for Beijing, China, was calculated based upon video observations and for Lahore, Pakistan, vehicle occupancy was determined empirically. As many people prefer to drive their own private cars, further congestion might be caused. It should be noted that, in developing countries like Pakistan and India, not many people can afford a car. There is also a trend of drivers sitting in front seats and owners sitting at the back of cars. As a result, the average vehicle occupancy rate in these two countries is more than that in other countries. Furthermore, no school buses are operating in Pakistan. The majority of the schoolto-home and home-to-school trips are made on private cars. People who can afford and use cars in day-to-day activities for work and recreational purposes rarely use public transportation for the commute. Thus, all of these reasons worsen the already depreciated transportation networks.

Our research shows that if autonomous micro cars are deployed in parallel with autonomous cars or manual cars, the road capacity can be increased vastly as per the results of this simulation model. Furthermore, manual micro cars despite their significant reaction delay can also increase the capacity of any road network [2]. Thus, where average vehicle occupancy is less than 2 and congestion is high, the notion of manual/autonomous micro cars should be adopted to increase the traffic flow rate. The ability for these vehicles to meet the demands of consumers and transport systems in emerging market, especially in dense megacities, needs new regulatory standards that take into account the different operating environments and demands of such vehicles.

\section{Conclusions}

In this paper, we study the heterogeneity in traffic by comparing different penetration rates of six different types of vehicles, including autonomous cars, autonomous buses, autonomous micro cars, manual cars, manual buses, and manual micro cars. A modified cellular automata model was developed to simulate real-time traffic scenarios. The characteristics and parameters of all six vehicles were established into the model. Additionally, two sets of lanechanging behavior (aggressive lane changing and polite lane changing) were merged in the model, assuming that manual vehicles can only follow aggressive lane-change behavior, while autonomous vehicles can follow both. Different compositions of six vehicles were simulated to understand the impact of different vehicle modes on overall traffic flow.

The main findings of this study are summarised as follows:

(i) Polite lance change behavior does not significantly affect the network's capacity as only autonomous vehicles are allowed to lane-change in a polite manner that requires certain conditions to be met. Since both manual and autonomous vehicles can change lanes aggressively when required, the capacity of network increases with the increase in ALC.

(ii) With the increase of penetration percentage of autonomous cars, autonomous buses, micro autonomous cars and micro manual cars, the overall flow rate of the network increases thus demonstrating a noteworthy increase in capacity. In the congestion phase, either manual or autonomous, micro cars can reduce traffic congestion and delays due to their smaller size as they can change lanes more effectively than conventional vehicles.

(iii) If average vehicle occupancy is less than 2, then autonomous micro cars and manual micro cars will immensely abate the traffic congestion and can provide more stability in the congestion phase.

In summary, although standard autonomous vehicles could increase the traffic flow rate, autonomous micro cars have the potential to raise it further and reduce congestion significantly when coupled with standard autonomous vehicles. Unlike the previous studies that focused on the penetration rate of standard autonomous cars, this study gives a different perspective by introducing different modes of autonomous vehicles, which can better describe realworld traffic. The finding of this study provides an understanding of the influence of autonomous vehicles on heterogeneous traffic flow. Future work can be extended to model different traffic scenarios. The impact of different types of autonomous vehicles on network-wide traffic performance can be investigated through traffic assignment. In addition, the influence of micro autonomous cars on parking as well as ridesharing should be explored.

\section{Data Availability}

For this study, simulations were ran and then data were generated.

\section{Conflicts of Interest}

The authors declare that they have no conflicts of interest.

\section{Acknowledgments}

This research was funded by the "Research of Transportation Strategy for Powerful Nation" and the Major Consulting Project of the Chinese Academy of Engineering (No. 2017ZD07), and special gratitude shall be extended to Professor Ruimin Li, Professor Xiang Zhang, Mr. $\mathrm{Hu} \mathrm{Li}$, Miss Shumaila Qadeer, Miss Anum Tanveer, Miss Faiza Naveed, and Miss Sadia for their invaluable suggestions.

\section{References}

[1] M. Richardson and G. Rose, "12th world conference on transport research," in Proceedings of the World Conference on Transport Research, Lisbon, Portugal, July 2010.

[2] R. Mu and T. Yamamoto, "An analysis on mixed traffic flow of conventional passenger cars and microcars using a cellular 
automata model," Procedia - Social and Behavioral Sciences, vol. 43, pp. 457-465, 2012.

[3] R. Mu and T. Yamamoto, "Analysis of micro-cars' influence on traffic network using a microscopic simulator," Journal of Transportation Systems Engineering and Information Technology, vol. 13, no. 6, pp. 44-51, 2013.

[4] M. W. Levin and S. D. Boyles, "A multiclass cell transmission model for shared human and autonomous vehicle roads," Transportation Research Part C: Emerging Technologies, vol. 62, pp. 103-116, 2016.

[5] Y. Liu, J. Guo, J. Taplin, and Y. Wang, "Characteristic analysis of mixed traffic flow of regular and autonomous vehicles using cellular automata," Journal of Advanced Transportation, vol. 2017, Article ID 8142074, 10 pages, 2017.

[6] F. Zhou, X. Li, and J. Ma, "Parsimonious shooting heuristic for trajectory design of connected automated traffic part I: theoretical analysis with generalized time geography," Transportation Research Part B: Methodological, vol. 95, pp. 394-420, 2017.

[7] T.-H. Chang and I. S. Lai, "Analysis of characteristics of mixed traffic flow of autopilot vehicles and manual vehicles," Transportation Research Part C: Emerging Technologies, vol. 5, no. 6, pp. 333-348, 1997.

[8] V. A. C. van den Berg and E. T. Verhoef, "Autonomous cars and dynamic bottleneck congestion: the effects on capacity, value of time and preference heterogeneity," Transportation Research Part B: Methodological, vol. 94, pp. 43-60, 2016.

[9] P. Tientrakool, Y. C. Ho, and N. F. Maxemchuk, "Highway capacity benefits from using vehicle-to-vehicle communication and sensors for collision avoidance," in Proceedings of the 2011 IEEE Vehicular Technology Conference (VTC Fall), September 2011.

[10] W. Brilon and N. Wu, "Evaluation of cellular automata for traffic flow simulation on freeway and urban streets," in Traffic and Mobility, pp. 163-180, Springer, Heidelberg, Germany, 1999.

[11] S. C. Lo and C. H. Hsu, "Analytical solution of traffic cellular automata model," in Proceedings of the AIP Conference Proceedings, Crete, Greece, American Institute of Physics, 2009.

[12] G. J. Horng, "Using cellular automata for parking recommendations in smart environments," PLOS one, vol. 9, no. 8, 2014.

[13] A. Talebpour, H. S. Mahmassani, F. Mete, and S. H. Hamdar, "Near-crash identification in a connected vehicle environment," Transportation Research Record: Journal of the Transportation Research Board, vol. 2424, no. 1, pp. 20-28, 2014.

[14] I. G. Jin and G. Orosz, "Dynamics of connected vehicle systems with delayed acceleration feedback," Transportation Research Part C: Emerging Technologies, vol. 46, pp. 46-64, 2014.

[15] R. Wang, Y. Li, and D. B. Work, "Comparing traffic state estimators for mixed human and automated traffic flows," Transportation Research Part C: Emerging Technologies, vol. 78, pp. 95-110, 2017.

[16] M. Green, "“How long does it take to stop?" methodological analysis of driver perception-brake times," Transportation Human Factors, vol. 2, no. 3, pp. 195-216, 2000.

[17] V. V. Dixit, S. Chand, and D. J. Nair, "Autonomous vehicles: disengagements, accidents and reaction times," PLoS One, vol. 11, no. 12, 2016.

[18] X. Qu, M. Yang, F. Yang, B. Ran, and L. Li, “An improved single-lane cellular automaton model considering driver's radical feature," Journal of Advanced Transportation, vol. 2018, Article ID 3791820, 10 pages, 2018.

[19] T. Wietholt and J. Harding, "Influence of dynamic traffic control systems and autonomous driving on motorway traffic flow," Transportation research procedia, vol. 15, pp. 176-186, 2016.

[20] M. Gouy, Behavioural Adaption of Drivers of Unequipped Vehicles to Short Time Headways Observed in a Vehicle Platoon, Ph.D. Thesis, The Open University, Milton Keynes, UK, , 2013.

[21] J. Vander Werf, S. E. Shladover, M. A. Miller, and N. Kourjanskaia, "Effects of adaptive cruise control systems on highway traffic flow capacity," Transportation Research Record: Journal of the Transportation Research Board, vol. 1800, no. 1, pp. 78-84, 2002.

[22] T. Muhammad, F. A. Kashmiri, H. Naeem, X. Qi, H. ChiaChun, and H. Lu, "Simulation study of autonomous vehicles' effect on traffic flow characteristics including autonomous buses," Journal of Advanced Transportation, vol. 2020, Article ID 4318652, 17 pages, 2020.

[23] M. Tanveer, F. A. Kashmiri, H. Naeem et al., "An assessment of age and gender characteristics of mixed traffic with autonomous and manual vehicles: a cellular automata approach," Sustainability, vol. 12, no. 7, p. 2922, 2020.

[24] F. T. Sparrow and R. K. Whitford, "The coming mini/micro car crisis: do we need a new definition?" Transportation Research Part A: General, vol. 18, no. 4, pp. 289-303, 1984.

[25] R. Mu and T. Yamamoto, "Analysis of the safety and environmental effects of introducing microcars into traffic flows," in Proceedings of the Transportation Research Board Annual Meeting, Washington, DC, USA, 2013.

[26] P. G. Gipps, "A model for the structure of lane-changing decisions," Transportation Research Part B: Methodological, vol. 20, no. 5, pp. 403-414, 1986.

[27] Q. Yang and H. N. Koutsopoulos, "A microscopic traffic simulator for evaluation of dynamic traffic management systems," Transportation Research Part C: Emerging Technologies, vol. 4, no. 3, pp. 113-129, 1996.

[28] P. Hidas, "Modelling vehicle interactions in microscopic simulation of merging and weaving," Transportation Research Part C: Emerging Technologies, vol. 13, no. 1, pp. 37-62, 2005.

[29] A. Kesting, M. Treiber, and D. Helbing, "General lanechanging model MOBIL for car-following models," Transportation Research Record: Journal of the Transportation Research Board, vol. 1999, no. 1, pp. 86-94, 2007.

[30] Z. Zheng, "Recent developments and research needs in modeling lane changing," Transportation Research Part B: Methodological, vol. 60, pp. 16-32, 2014.

[31] T. Toledo, C. F. Choudhury, and M. E. Ben-Akiva, "Lanechanging model with explicit target lane choice," Transportation Research Record: Journal of the Transportation Research Board, vol. 1934, no. 1, pp. 157-165, 2005.

[32] J. Yuan, M. Abdel-Aty, Q. Cai, and J. Lee, "Investigating drivers' mandatory lane change behavior on the weaving section of freeway with managed lanes: a driving simulator study," Transportation Research Part F: Traffic Psychology and Behaviour, vol. 62, pp. 11-32, 2019.

[33] L. Fei, H. B. Zhu, and X. L. Han, "Analysis of traffic congestion induced by the work zone," Physica A: Statistical Mechanics and Its Applications, vol. 450, pp. 497-505, 2016.

[34] S. W. Kim and W. Liu, "Cooperative autonomous driving: a mirror neuron inspired intention awareness and cooperative perception approach," IEEE Intelligent Transportation Systems Magazine, vol. 8, no. 3, pp. 23-32, 2016. 
[35] FHWA, Average Vehicle Occupancy Factors for Computing Travel Time Reliability Measures and Total Peak Hour Excessive Delay Metrics, F.H.A. (FHWA), 2018.

[36] Average Car and Van Occupancy in England from 2002 to 2018, statista, December 2020.

[37] "What's happening with car occupancy?" in Charting Transport Looking at the Transport through Graphs and Maps, April 2016.

[38] B. Sudhakara Reddy and P. Balachandra, "Urban mobility: a comparative analysis of megacities of India," Transport Policy, vol. 21, pp. 152-164, 2012.

[39] 2009 Canadian Vehicle Survey Summary Report, 2009.

[40] European Environment Agency, In Occupancy Rates of Passenger Vehicles, European Environment Agency, København, Denmark, 06 August 2015. 